Research Article

\title{
Mixed Convection of a Radiating Magnetic Nanofluid past a Heated Permeable Stretching/Shrinking Sheet in a Porous Medium
}

\author{
Feleke Buta Tadesse $\mathbb{D}^{1},{ }^{1}$ Oluwole Daniel Makinde $\mathbb{C D}^{2},{ }^{2}$ and Lemi Guta Enyadene ${ }^{1}$ \\ ${ }^{1}$ Adama Science and Technology University, Department of Applied Mathematics, Adama, Ethiopia \\ ${ }^{2}$ Faculty of Military Science Stellenbosch University, Stellenbosch, Western Cape, South Africa \\ Correspondence should be addressed to Feleke Buta Tadesse; feleke.buta@astu.edu.et
}

Received 18 November 2020; Revised 16 February 2021; Accepted 11 May 2021; Published 2 June 2021

Academic Editor: Amer Rasheed

Copyright (c) 2021 Feleke Buta Tadesse et al. This is an open access article distributed under the Creative Commons Attribution License, which permits unrestricted use, distribution, and reproduction in any medium, provided the original work is properly cited.

\begin{abstract}
This paper analyzes the collective effects of buoyancy force, thermal radiation, convective heating, and magnetic field on stagnation point flow of an electrically conducting nanofluid past a permeable stretching/shrinking sheet in a porous medium. Similarity transformations are used on the resulting nonlinear partial differential equations to transfer into a system of coupled nonlinear ordinary differential equations. The fourth-fifth-order Runge-Kutta-Fehlberg method with shooting technique is applied to solve numerically. Results are obtained for dimensionless velocity, temperature, and nanoparticle volume fraction as well as the skin friction and local Nusselt and Sherwood numbers. The results indicate the existence of two real solutions for the shrinking sheet in the range of $\lambda_{c}<\lambda<0$. The fluid flow stability is maintained by increasing the magnetic field effect, whereas the porous medium parameter inflates the flow stability. It is also noted that both the skin friction coefficient and the local Sherwood number approximately decline with the intensification of thermal radiation within the range from $9.83 \%$ to $14 \%$ and the range from $48.86 \%$ to $78.66 \%$, respectively. It is also evident in the present work that the local Nusselt number upsurges with the porous and suction/injection parameters.
\end{abstract}

\section{Introduction}

Problems of fluid flow and heat transfer in a porous medium have a wide range of applications in various engineering systems. These problems occur in the storage of radioactive nuclear waste, transpiration cooling, separation processes in chemical industries, filtration processes, transport processes in aquifers, groundwater pollution, geothermal extraction, and fiber insulation as reported by [1]. Using nanofluid is a potential technique to improve the problem of heat transfer in high-technology industries as mentioned by [2]. The author of [3] is the leading person to specify engineered colloids consisting of nanoparticles dispersed in a base fluid like water, ethylene glycol, engine oil, or others as a nanofluid. On the other hand, using porous media is an effective method for heat transfer enhancement in industrial systems as described by [4]. Following these facts, a lot of work has been done on fluid flow and heat transfer problems in a porous medium with nanofluid. Accordingly, [5] pointed out that an alternative method for improving heat transfer characteristics in various thermal devices is using porous medium and nanofluid together. Reference [6] conducted a wide-ranging review of the simultaneous application of nanofluids and porous media for heat transfer enhancement cases in thermal systems with diverse conditions. Different aspects of this problem have been explored by many investigators such as those in [7-11].

Fluid flow caused by a stretching/shrinking sheet has many practical applications in the fields of metallurgy, polymer technology, chemical engineering, and so forth as explained by $[12,13]$. The study of magnetohydrodynamic (MHD) stagnation point flow past a stretching/shrinking 
sheet has been carried out by many researchers in various ways due to its practical applications. A few examples are [14-23]. Furthermore, [24] numerically studied magnetohydrodynamic (MHD) flow of an electrically conducting fluid past a stretching sheet in a porous medium with a buoyancy force effect. It was reported that the solute buoyancy effect and electric field override the fluctuations in inertia and viscous forces to enhance the velocity field. Reference [25] theoretically examined unsteady mixed convection boundary-layer flow of a heated vertical surface in a nanofluid-saturated porous medium. The result therein indicated that as the values of the thermophoresis and Brownian motion parameters increase, the local skin friction coefficient increases, whereas the Nusselt number decreases. Moreover, the Sherwood number increases as the thermophoresis parameter increases and decreases as the Brownian motion parameter increases.

Mixed convection of heat and mass transfer has different applications in heat exchanges, solar collectors, nuclear reactors, atmospheric boundary layer flow, nanotechnology, and electronic apparatus as reported by [26]. Besides being used as a convective heat transfer mechanism, thermal radiation plays a key role in managing heat transfer processes. Controlling the effects of radiation in fluid flow and heat transfer problems has vigorous significance in engineering processes, nuclear plants, space vehicles, gas turbines, and satellites involving high temperatures needed in designing specific equipment as reported by [27, 28]. Taking this fact into account, various researchers have investigated radiation effects on mixed convection flow problems in view of different aspects of the problem. Reference [29] numerically investigated hydromagnetic mixed convection stagnation point flow past a vertical plate embedded in a highly porous medium with the effects of radiation and internal heat generation. The result therein revealed that the local skin friction, local Nusselt number, and local Sherwood number intensify as magnetic field strength and radiation parameters increase. Reference [30] investigated steady two-dimensional magnetohydrodynamic (MHD) flow past a permeable stretching or shrinking sheet with radiation effects. According to the result therein, dual solutions exist for shrinking surfaces, and the solution is unique for the stretching case. Reference [31] analyzed the heat and mass transfer characteristics in a two-dimensional stagnation point flow over a stretching vertical sheet under effects of buoyancy force and thermal radiation using the implicit finite-difference method. Extending the idea, [32] numerically investigated buoyancy and thermal radiation effect on heat transfer of water-based nanofluid flow over a stretching sheet. The result therein showed that the skin friction coefficient upturns with the intensification of the magnetic field and the radiation effect and declines with buoyancy parameter and nanoparticle volume fraction parameter.

Even though several works have been testified on fluid flow and heat transfer problems with nanofluid, there seem to be no efforts in the literature to consider the collective effects of buoyancy force, thermal radiation, viscous and porous dissipation, and porous medium on hydromagnetic stagnation point flow of nanofluid flow past a permeable stretching/shrinking sheet with convective boundary conditions.

Thus, our main objective is to analyze the mixed convection stagnation point flow of a radiating magnetic nanofluid over a permeable stretching/shrinking sheet in a porous medium with convective conditions. Moreover, the inclusion of viscous and porous dissipation terms in the energy equation enables us to examine their effect on fluid flow and heat transfer. Such magnetohydrodynamic (MHD) boundary layer fluid flows of an electrically conducting fluid past a stretching/shrinking sheet have various applications in modern metallurgical and metal-working processes such as drawing of continuous filaments through quiescent fluids and annealing and tinning of copper wires as mentioned in $[33,34]$. The present work is an extension from [15] to include the collective effects of thermal radiation, the permeability of the stretching/shrinking sheet, and porous medium. The similarity transformation technique is applied to transform the resulting system of partial differential equations into a system of nonlinear ordinary differential equations. The resulting system of nonlinear ordinary differential equations is solved using the fourth-fifth-order Runge-Kutta-Fehlberg method with shooting technique. The effects of various parameters on the velocity, temperature, and nanoparticle concentration are presented graphically, and skin friction coefficient, heat, and mass transfer rates are discussed quantitatively.

\section{Mathematical Formulation and Analysis}

We consider a steady, laminar, viscous, and incompressible two-dimensional stagnation point flow of a magnetic nanofluid past a heated permeable stretching/shrinking sheet which is embedded in a porous medium along $x$-direction. It is assumed that a constant magnetic field of strength $B_{0}$ is applied in the positive $y$-direction normal to the surface. The induced magnetic field is assumed to be small compared to the applied magnetic field. The ambient temperature of the fluid and the ambient concentration are taken as $T_{\infty}$ and $\phi_{\infty}$, respectively, while the surface below the stretching/shrinking sheet is heated by convection from a hot fluid having initial temperature $T_{f}$ with nanoparticles concentration $\phi_{f}$, which provides a heat transfer coefficient $h_{1}$ and mass transfer coefficient $h_{2}$. It is assumed that the porous medium is homogeneous and isotropic and saturated with a nanofluid which is in local thermal equilibrium with the solid matrix. Further, the flow is assumed to be slow so that a Forchheimer quadratic drag term does not appear in the momentum equation. The effects of thermal radiation are incorporated through energy equation. The fluid is considered to be a gray, absorbing emitting radiation but nonscattering medium and the Rosseland approximation is used to describe the radiative heat flux in the energy equation. We choose the coordinate system so that $x$-axis is along the stretching/shrinking sheet and $y$-axis is normal to the sheet surface. A sketch of the physical model and the coordinates are given in Figure 1.Using Buongiorno convective transport equations with the above stated physical situations, Oberbeck-Boussinesq, and the boundary layer 


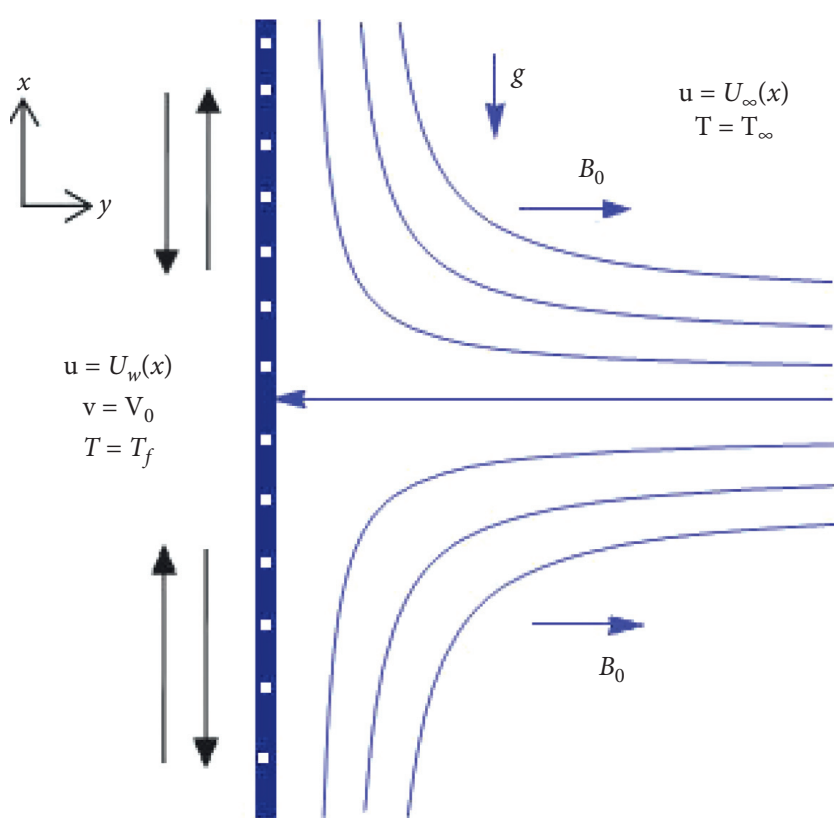

Figure 1: Flow diagram of the model.

approximation, the governing equations of the conservation of total mass, momentum, energy, and nanoparticles volume fraction in the presence of magnetic field past a permeable stretching/shrinking sheet take the following forms ([35-37]):

$$
\begin{aligned}
\frac{\partial u}{\partial x}+\frac{\partial v}{\partial y}= & 0 \\
u \frac{\partial u}{\partial x}+v \frac{\partial u}{\partial y}= & U_{\infty} \frac{\partial U_{\infty}}{\partial x}+\frac{\mu_{f}}{\rho_{f}} \frac{\partial^{2} u}{\partial y^{2}}-\frac{\sigma B_{0}^{2}}{\rho_{f}}\left(u-U_{\infty}\right)-\frac{\mu_{f}}{\rho_{f} K}\left(u-U_{\infty}\right) \\
& +\frac{1}{\rho_{f}}\left[\left(1-\phi_{\infty}\right) \rho_{f \infty} \beta g\left(T-T_{\infty}\right)-\left(\rho_{p}-\rho_{\infty}\right) g\left(\phi-\phi_{\infty}\right)\right] \\
u \frac{\partial T}{\partial x}+v \frac{\partial T}{\partial y}= & \alpha_{f}\left(1+\frac{16 \sigma^{*} T_{\infty}^{3}}{3 \mathrm{kk}^{*}}\right)\left(\frac{\partial^{2} T}{\partial y^{2}}\right)+\tau\left(D_{B} \frac{\partial \phi}{\partial y} \frac{\partial T}{\partial y}+\frac{D_{T}}{T_{\infty}}\left(\frac{\partial T}{\partial y}\right)^{2}\right) \\
& +\frac{\alpha_{f} \mu_{f}}{k}\left(\frac{\partial u}{\partial y}\right)^{2}+\left(\frac{\alpha_{f} \sigma B_{0}^{2}}{k}+\frac{\alpha_{f} \mu_{f}}{\mathrm{kK}}\right)\left(u-U_{\infty}\right)^{2}, \\
u \frac{\partial \phi}{\partial x}+v \frac{\partial \phi}{\partial y}= & D_{B} \frac{\partial^{2} \phi}{\partial y^{2}}+\left(\frac{D_{T}}{T_{\infty}}\right)\left(\frac{\partial^{2} T}{\partial y^{2}}\right),
\end{aligned}
$$

where the velocity components along the $x$-direction and $y$-direction are given by $u$ and $v$, respectively. The nanoparticle density, the density of base fluid, the absolute viscosity of the base fluid, the thermal diffusivity of the base fluid, the Stefan-Boltzmann constant, and the mean absorption coefficient are dented by $\rho_{p}, \rho_{f}, \mu_{f}, \alpha_{f}, \sigma^{*}$, and $k^{*}$, respectively. Furthermore, $\tau=\left(\rho_{c}\right)_{p} /\left(\rho_{c}\right)_{f}$ is the ratio of nanoparticle heat capacity and the base fluid heat capacity, $K$ is the porous medium permeability, $T$ is the local temperature, $\phi$ is the local solid volume fraction of the nanofluid, $\beta$ is volumetric thermal expansion coefficient of the base fluid, $D_{B}$ is the Brownian diffusion coefficient, $D_{T}$ is the thermophoretic diffusion coefficient, and $\mathrm{g}$ is the acceleration due to gravity. The subscript $\infty$ denotes the values at large values of $y$ where the fluid is quiescent. The boundary conditions are written as 


$$
\begin{aligned}
y & =0: u=\mathrm{ax}, v=V_{0},-k \frac{\partial T}{\partial y}=h_{1}\left(T_{f}-T\right), \\
-D_{B} \frac{\partial \phi}{\partial y} & =h_{2}\left(\phi_{f}-\phi\right), \\
y & \longrightarrow \infty: u \longrightarrow U_{\infty}(x), T \longrightarrow T_{\infty}, \phi \longrightarrow \phi_{\infty},
\end{aligned}
$$

where $U_{\infty}(x)=\left(\alpha_{f} / x\right) \mathrm{Ra}_{x}^{1 / 2}$ is the external velocity, $\mathrm{Ra}_{x}=$ $(1-\phi) g \beta\left(T_{f}-T_{\infty}\right) x^{3} / \nu \alpha_{f}$ is the local Rayleigh number, $U_{w}(x)=$ ax is the stretching/shrinking velocity of the sheet, $a$ is a constant, and $V_{0}$ is the wall mass transfer velocity.

We introduce the following nondimensional similarity variables in order to transfer the governing equations (A.1)-(A.4) and boundary equations (A.5)-(A.6):

$$
\begin{aligned}
\eta & =\frac{y}{x} \mathrm{Ra}_{x}^{1 / 4}, \psi(x, y)=\alpha_{f} \mathrm{Ra}_{x}^{1 / 4} s(\eta), \\
\theta(\eta) & =\frac{T-T_{\infty}}{T_{f}-T_{\infty}}, f(\eta)=\frac{\phi-\phi_{\infty}}{\phi_{f}-\phi_{\infty}} .
\end{aligned}
$$

The equation of continuity is satisfied for the chosen stream function $\psi(x, y)$ such that

$$
\begin{gathered}
u=\frac{\partial \psi}{\partial y} \\
v=-\frac{\partial \psi}{\partial x} .
\end{gathered}
$$

Now using the transformation quantities, we obtained transformed nonlinear ordinary differential equations as follows:

$$
\begin{gathered}
s^{\prime \prime \prime}-\left(M+\frac{1}{\mathrm{Da}}\right)\left(s^{\prime}-1\right)+\frac{1}{4 \operatorname{Pr}}\left[3 s s^{\prime \prime}-2 s^{\prime 2}+2\right]+\theta-\mathrm{Nrf}=0 \\
\left(1+\frac{4}{3} R\right) \theta^{\prime \prime}+\frac{3}{4} s \theta^{\prime}+\operatorname{Ec} \operatorname{Pr}\left[s^{\prime \prime 2}+\left(M+\frac{1}{\mathrm{Da}}\right)\left(s^{\prime}-1\right)^{2}\right]+\mathrm{Nbf}^{\prime} \theta^{\prime}+\mathrm{Nt} \theta^{\prime 2}=0 \\
f^{\prime \prime}+\frac{\mathrm{Nt}}{\mathrm{Nb}} \theta^{\prime \prime}+\frac{3}{4} \mathrm{Lef}^{\prime} s=0
\end{gathered}
$$

Here, primes denote differentiation with respect to $\eta$ and $s^{\prime}, \theta, f$, and $\eta$ are the dimensionless velocity, temperature, particle concentration, and similarity variable, respectively. The variables $\mathrm{Nr}, \mathrm{Pr}$, Le, $\mathrm{Nb}, \mathrm{Nt}, R, M, \mathrm{Ec}, \mathrm{Da}, \delta$, and $\lambda$ denote the nanofluid buoyancy ratio parameter, Prandtl number, the Lewis number, the Brownian motion parameter, the thermophoresis diffusion parameter, the radiation parameter, magnetic field parameter, Eckert number, the porous media parameter, suction/injection parameter, and stretching/shrinking parameter, respectively. The parameters and dimensionless numbers are defined as follows:

$$
\begin{gathered}
\mathrm{Nr}=\frac{\left(\rho_{p}-\rho_{f}\right)\left(\phi_{w}-\phi_{\infty}\right)}{\rho_{f} \beta\left(1-\phi_{w}\right)\left(T_{f}-T_{\infty}\right)}, \mathrm{Nb}=\frac{\tau D_{B}\left(\phi_{w}-\phi_{\infty}\right)}{\alpha_{f}}, \mathrm{Nt}=\frac{\tau D_{T}\left(T_{f}-T_{\infty}\right)}{\alpha_{f} T_{\infty}}, \\
M=\frac{\sigma_{f} B_{0}^{2} x^{2}}{\mu_{f} \mathrm{Ra}_{x}^{1 / 2}}, \mathrm{Ec}=\frac{U_{\infty}^{2}}{C_{p f}\left(T_{f}-T_{\infty}\right)}, \mathrm{Nc}=\frac{h_{1} x}{\mathrm{kRa}_{x}^{1 / 4}}, \mathrm{Nc}^{*}=\frac{h_{2} x}{D_{B} \mathrm{Ra}_{x}^{1 / 4}} \mathrm{Le}=\frac{\alpha_{f}}{D_{B}}, \\
R=\frac{4 \sigma^{*} T_{\infty}^{3}}{k^{*} k}, \mathrm{Da}=\frac{\mathrm{KRa}_{x}^{1 / 2}}{x^{2}}, \lambda=\frac{\mathrm{ax}^{2}}{\alpha_{f} \sqrt{\mathrm{Ra}_{x}}}, \delta=\frac{-4 x}{3 \alpha_{f} \mathrm{Ra}_{x}^{1 / 4}} V_{0}, \operatorname{Pr}=\frac{\nu}{\alpha_{f}} .
\end{gathered}
$$

The boundary conditions in terms of the new variables become

$$
\begin{aligned}
& \eta=0: s(0)=\delta, s^{\prime}(0)=\lambda, \theta^{\prime}(0)=-\mathrm{Nc}[1-\theta(0)], \\
& f^{\prime}(0)=-\mathrm{Nc}^{*}[1-f(0)] \text {, } \\
& \eta \longrightarrow \infty: s^{\prime}(\infty)=1, \theta(\infty)=0, f(\infty)=0 \text {. }
\end{aligned}
$$

where $\mathrm{Nc}$ and $\mathrm{Nc}^{*}$ are the heat transfer convective parameter and the mass transfer convective parameter, respectively. When $\mathrm{Nc} \longrightarrow \infty$ and $\mathrm{Nc}^{*} \longrightarrow \infty$, the convective boundary conditions reduce to a uniform surface temperature and nanoparticle concentration.

The important physical quantities of interest, in this problem, are the dimensionless skin friction coefficient, the local Nusselt number, and the local Sherwood number. Skin friction coefficient measures the friction that occurs at the 
boundary, Nusselt number helps to determine the wall heat flux, and Sherwood number is used to determine the wall mass flux as explained by [38]. These important engineering parameters are defined as

$$
\begin{aligned}
s^{\prime \prime}(0) & =\frac{C_{f} x^{2}}{\mu \alpha_{f} \mathrm{Ra}_{x}^{3 / 4}}, \\
\mathrm{Nu}_{x} & =\frac{\mathrm{xq}_{w}^{\prime \prime}}{k\left(T_{f}-T_{\infty}\right)}, \\
\mathrm{Sh}_{x} & =\frac{\mathrm{xq}_{m}^{\prime \prime}}{D_{B}\left(\phi_{w}-\phi_{\infty}\right)},
\end{aligned}
$$

where $\tau_{w}$ is the wall shear stress, $q_{w}^{\prime \prime}$ is the wall heat flux due to the temperature gradient, and $\mathrm{q}_{m}^{\prime \prime}$ is the wall mass flux due to the Brownian motion force at $y=0$, and they are given by

$$
\begin{aligned}
& \tau_{w}=\mu\left(\frac{\partial u}{\partial y}\right) \\
& q_{w}^{\prime \prime}=-\left[\left(k+\frac{16 \sigma^{*} T_{\infty}^{3}}{3 k^{*}}\right) \frac{\partial T}{\partial y}\right], \\
& q_{m}^{\prime \prime}=-D_{B}\left(\frac{\partial \phi}{\partial y}\right) .
\end{aligned}
$$

In dimensionless form, the reduced local Nusselt and Sherwood numbers can be rewritten as

$$
\mathrm{Nu}=\mathrm{Nu}_{x} \mathrm{Ra}_{x}^{-1 / 4}=-\left(1+\frac{4}{3} R\right) \theta^{\prime}(0), \mathrm{Sh}=\mathrm{Sh}_{x} \mathrm{Ra}_{x}^{-1 / 4}=-f^{\prime}(0),
$$

where $\mathrm{Ra}_{x}, \mathrm{Nu}_{x}, \mathrm{Sh}_{x}$ are local Rayleigh number, local Nusselt number, and local Sherwood number, respectively.

\section{Numerical Procedures}

The fourth-fifth-order Runge-Kutta-Fehlberg method along with the shooting technique is implemented to analyze the flow model for the above coupled nonlinear ordinary differential equations (5)-(7) subject to the boundary conditions, as well as equations (9) and (10) for various values of the governing parameters. The solution procedure is an efficient, adaptive, and accurate method of order 4 with an error estimator of order 5. This method has been used by several researchers for solving problems of convective boundary layer flows for instance papers by [39]. To solve numerically using the Runge-Kutta-Fehlberg method, first, we need to reduce the coupled boundary value problem into a set of first-order ODEs to obtain a system of seven simultaneous equations of the first order.

Defining new variables, we obtain the following system:

$$
\begin{aligned}
s= & f_{1}, s^{\prime}=f_{2}, s^{\prime \prime}=f_{3}, \theta=f_{4}, \theta^{\prime}=f_{5}, f=f_{6}, f^{\prime}=f_{7}, \\
f_{1}^{\prime}= & f_{2}, \\
f_{2}^{\prime}= & f_{3}, \\
f_{3}^{\prime}= & \left(M+\frac{1}{\mathrm{Da}}\right)\left(f_{2}-1\right)-\frac{1}{4 \mathrm{Pr}}\left[3 f_{1} f_{3}-2 f_{2}^{2}+2\right]-f_{4}+\mathrm{Nrf}_{6}, \\
f_{4}^{\prime}= & f_{5}, \\
f_{5}^{\prime}= & \frac{1}{(1+(4 / 3) R)}\left[\frac{-3}{4} f_{1} f_{5}-\operatorname{Ec} \operatorname{Pr}\left(f_{3}^{2}+\left(M+\frac{1}{\mathrm{Da}}\right)\left(f_{2}-1\right)^{2}\right)-\mathrm{Nbf}_{7} f_{5}-\mathrm{Ntf}_{5}^{2}\right], \\
f_{6}^{\prime}= & f_{7}, \\
f_{7}^{\prime}= & \frac{-N t}{\mathrm{Nb}(1+(4 / 3) R)}\left[-\frac{3}{4} f_{1} f_{5}-\operatorname{EcPr}\left(f_{3}^{2}+\left(M+\frac{1}{\mathrm{Da}}\right)\left(f_{2}-1\right)^{2}\right)-\mathrm{Nbf}_{7} f_{5}-\mathrm{Ntf}_{5}^{2}\right], \\
& -\frac{3}{4} \operatorname{Lef}_{7} f_{1} .
\end{aligned}
$$


Here, prime denotes the differentiation with respect to $\eta$ and the corresponding initial conditions are

$$
\left[\begin{array}{l}
f_{1}(0) \\
f_{2}(0) \\
f_{3}(0) \\
f_{4}(0) \\
f_{5}(0) \\
f_{6}(0) \\
f_{7}(0)
\end{array}\right]=\left[\begin{array}{c}
\delta \\
\lambda \\
u_{1} \\
u_{2} \\
-\mathrm{Nc}\left(1-u_{2}\right) \\
u_{3} \\
-\mathrm{Nc}^{*}\left(1-u_{3}\right)
\end{array}\right] .
$$

Here, we require that seven initial conditions but one initial condition in each of $s, \theta$, and $f$ be unknown. Thus, the values of the missing initial conditions $u_{1}, u_{2}$, and $u_{3}$ are approximated by using Newton-Raphson method until the boundary conditions at $\eta \longrightarrow \infty$ are satisfied.

To estimate the value of $\eta_{\infty}$, we start with some initial guess value and solve the problem consisting of equations (5)-(7). The process of finding a solution is repeated with another larger value of $\eta_{\infty}$ until two consecutive values of the missing boundary conditions at $\eta=0$ differ only after desired significant digits. The last value $\eta_{\infty}$ is taken as the finite value of the limit $\eta_{\infty}$ for the particular set of parameters. For our case, we choose $\eta_{\infty}=12$ so that the boundary conditions given by equation (10) become $s^{\prime}(12)=1, \theta(12)=0$, and $f(12)=0$. The choice of $\eta_{\infty}=12$ ensures that all numerical solutions approached the asymptotic values correctly. MAPLE software is used to generate the numerical solutions of the transformed equations and boundary conditions. The CPU time is 6.15 seconds on average for each computational result.

\section{Result and Discussion}

The combined effects of buoyancy force, thermal radiation, porous medium, convective heating, viscous dissipation, magnetic field, and nanofluid parameters on the dimensionless velocity, temperature, rescaled nanoparticle volume fraction, skin friction, and heat and mass transfer rates from a permeable stretching/shrinking sheet are investigated numerically and presented as follows.

4.1. Velocity Profiles with Parameter Variations. The alteration of the dimensionless velocity profile with the magnetic field and nanofluid buoyancy ratio parameters is depicted in Figure 2(a). As the magnetic field applied on the stretching surface increases, the velocity boundary layer thickness decreases. It is further noted that the parameter ratio of buoyancy forces due to the thermal expansion of the base fluid and difference in densities of the nanoparticles and the base fluid (water) amplifies the dimensionless velocity profile drops. We thus deduce that the flow in the upward direction decelerates as the nanofluid buoyancy force due to the difference in densities of the nanoparticles and the base fluid (water) toughens. The role of porous media and mass suction/injection parameters on the dimensionless velocity profile is portrayed through Figure 2(b). The dimensionless velocity profile drops with an increment in porous media parameter and the momentum boundary layer gets thickened as the porosity of the media increases. According to the numerical result obtained in Figure 2(b), an increase in the mass suction/injection parameter decelerates the dimensionless velocity profile within the momentum boundary layer. The reason for the deceleration of the fluid flow is that suction carries on the distance fluid into the region adjacent to the sheet, thereby enhancing the viscosity which in turn opposes the fluid motion.

Figure 3(a) shows the dimensionless velocity profile for different values of the stretching/shrinking parameter, while other parameters are assumed to be constant. As shown in this figure, when the stretching velocity increases, the dimensionless velocity profile decreases just after the transition point $\eta=0.56$ but, very near to the sheet surface, a reverse phenomenon is observed. The momentum boundary layer thickness is also dependent on the stretching/shrinking parameter. For the stretching sheet, the momentum boundary layer thickness is larger than the shrinking case for layers of the fluid far from the sheet surface starting at $\eta=0.56$. For highly shrinking surfaces, exaggerated change in dimensionless velocity and boundary layer thickness is observed, which is contrary to a stretching surface. These kinds of changes in dimensionless velocity and momentum boundary layer thickness can occur until the shrinking surface reaches the critical stretching/shrinking parameter $\left(\lambda_{c}\right)$ value. The convective parameter, Nc, measures the ratio of conduction resistance to convection resistance within the body. When $\mathrm{Nc}=0$, no convection takes place at the surface. As shown in Figure 3(b), the increment in convective parameter results in an increment in dimensionless velocity profile, and, at the same time, the momentum boundary layer thickness goes in a reverse manner.

4.2. Temperature Profiles with Parameter Variations. The variation of the dimensionless temperature profile with transverse distance is shown in Figures 4 and 5 for different values of the flow governing parameters. The effects of stretching/shrinking and magnetic field parameters on the dimensionless temperature profile within the thermal boundary layer are shown in Figure 4(a). In the absence of a magnetic field parameter, the dimensionless surface temperature is found to be lower and increases with an increasing magnetic field. This is because of the fact that a higher magnetic field produces a force called the Lorentz force which acts in the opposite direction of motion and hence overwhelms the velocity field. Simultaneously, it generates some thermal energy in the fluid motion, which causes a rise in the fluid temperature and its layer thickness. It is also important to note that the thermal boundary layer thickness decreases with increasing values of the stretching/ shrinking parameter.

Figure 4(b) illustrates the influence of the mass transfer and porous media parameters on the dimensionless temperature profiles. For a stretching problem, solutions exist for both mass suction and injection. For specified values of other parameters, the dimensionless surface temperature 


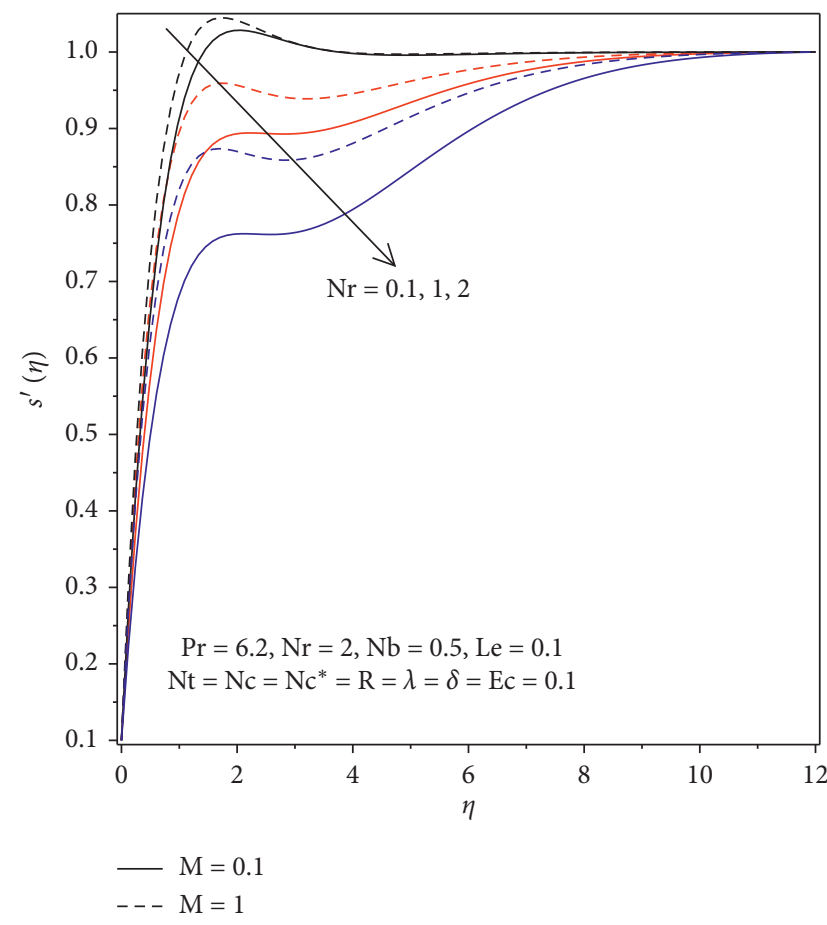

(a)

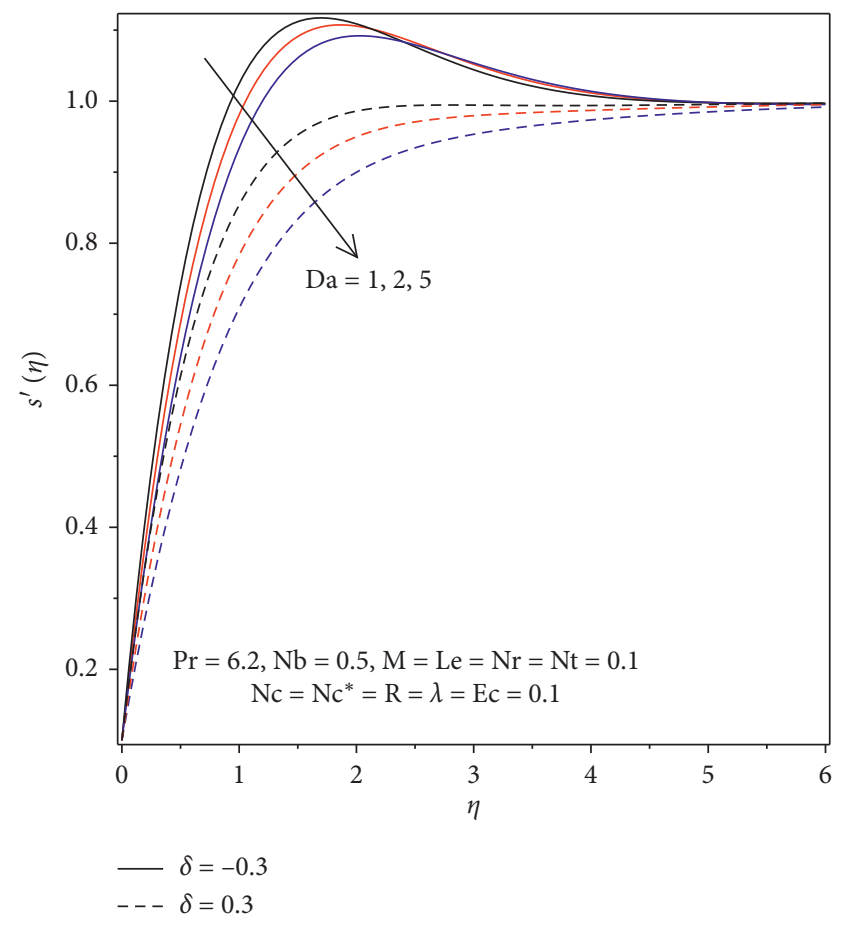

(b)

Figure 2: (a) Influence of $\mathrm{Nr}$ and $M$ on the velocity profile. (b) Influence of Da and $\delta$ on the velocity profile.

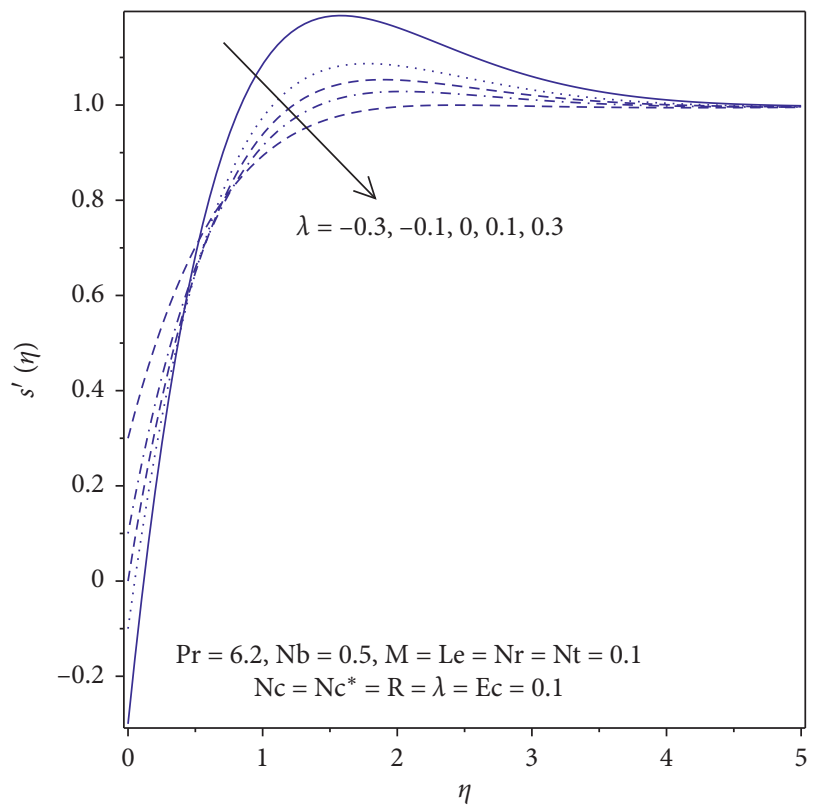

(a)

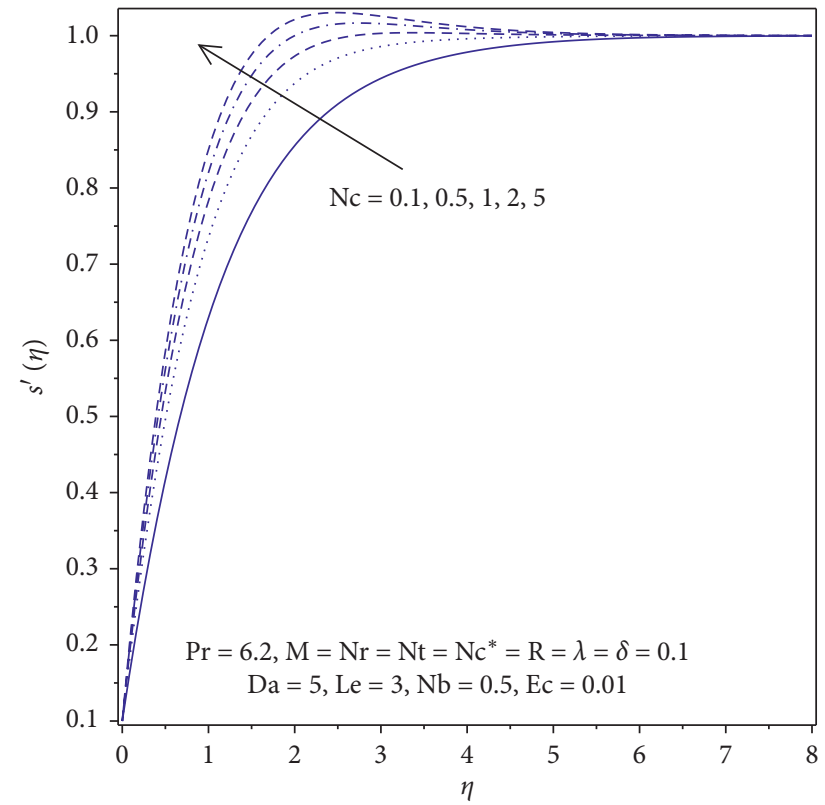

(b)

Figure 3: (a) Influence of $\lambda$ on the velocity profile. (b) Influence of Nc on the velocity profile.

reduces with increasing mass suction/injection parameter. The thermal boundary layer thickness becomes thicker into the fluid. The dimensionless surface temperature decreases as the porous media parameter increases. It is also noted that the thermal boundary layer thickness formed through injection exceeds thermal boundary layer thickness formed through suction for any porous medium.

As per the illustration in Figure 5(a), the dimensionless temperature profile increases as the nanofluid buoyancy ratio parameter increases. This is from the reality that 


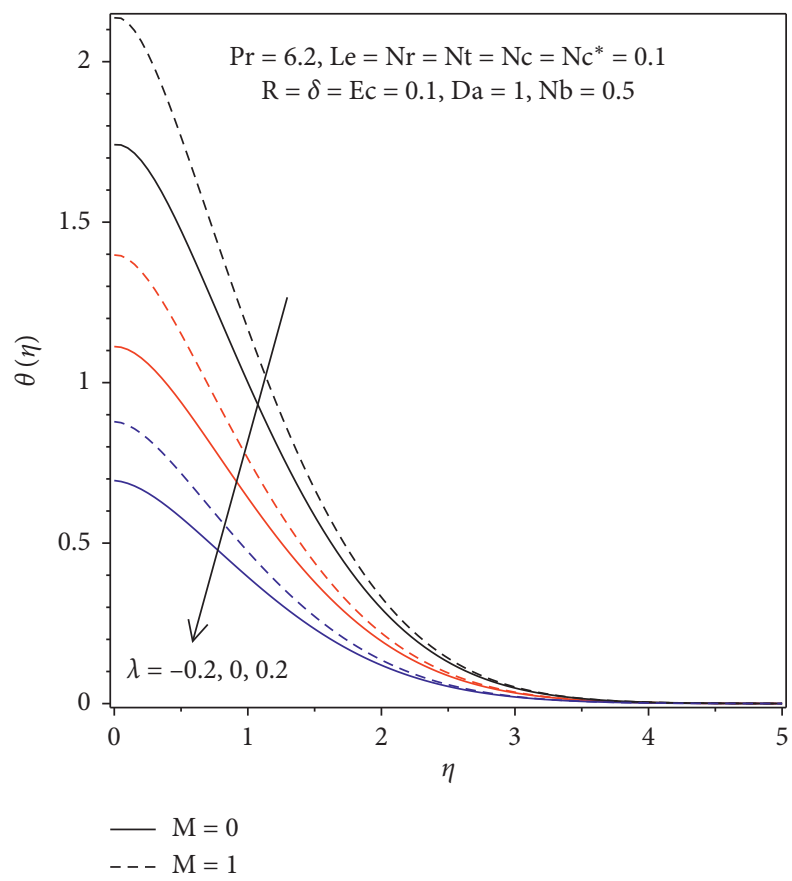

(a)

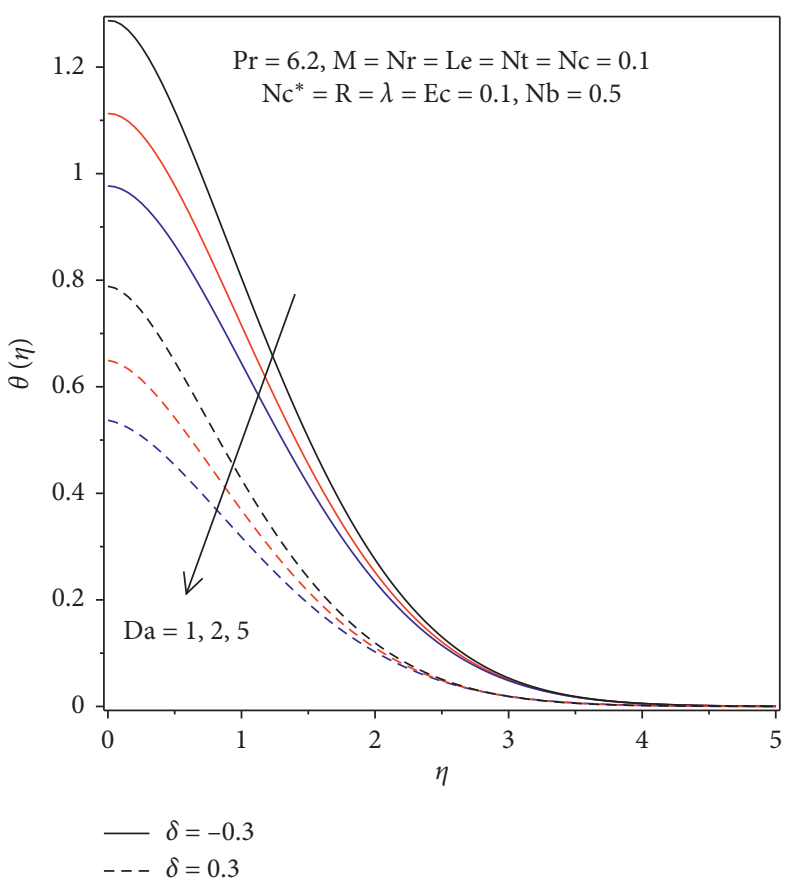

(b)

Figure 4: (a) Influence of $\lambda$ and $M$ on the temperature profile. (b) Influence of $\mathrm{Da}$ and $\delta$ on the temperature profile.

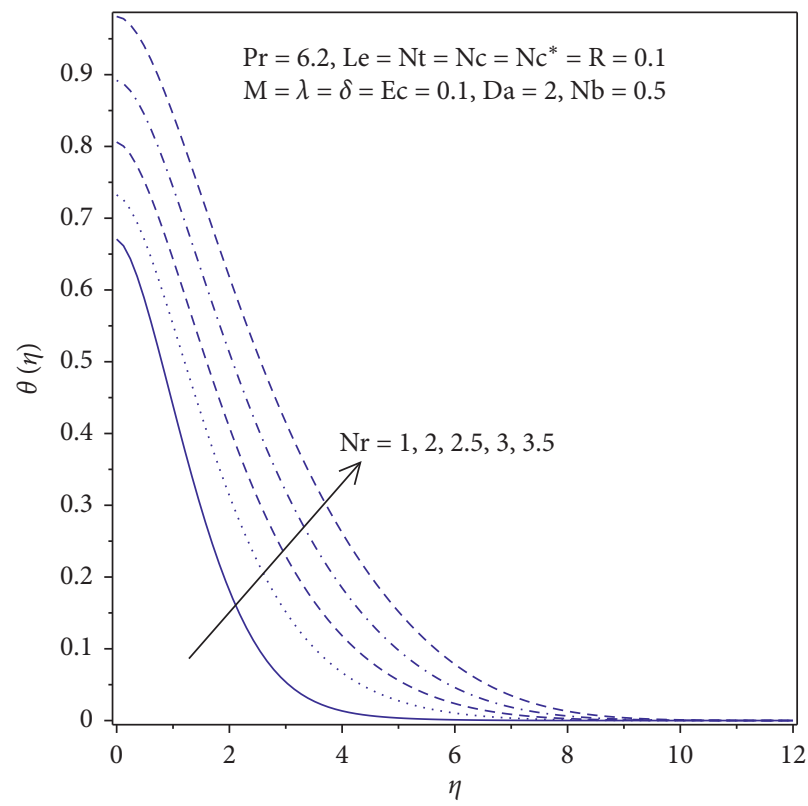

(a)

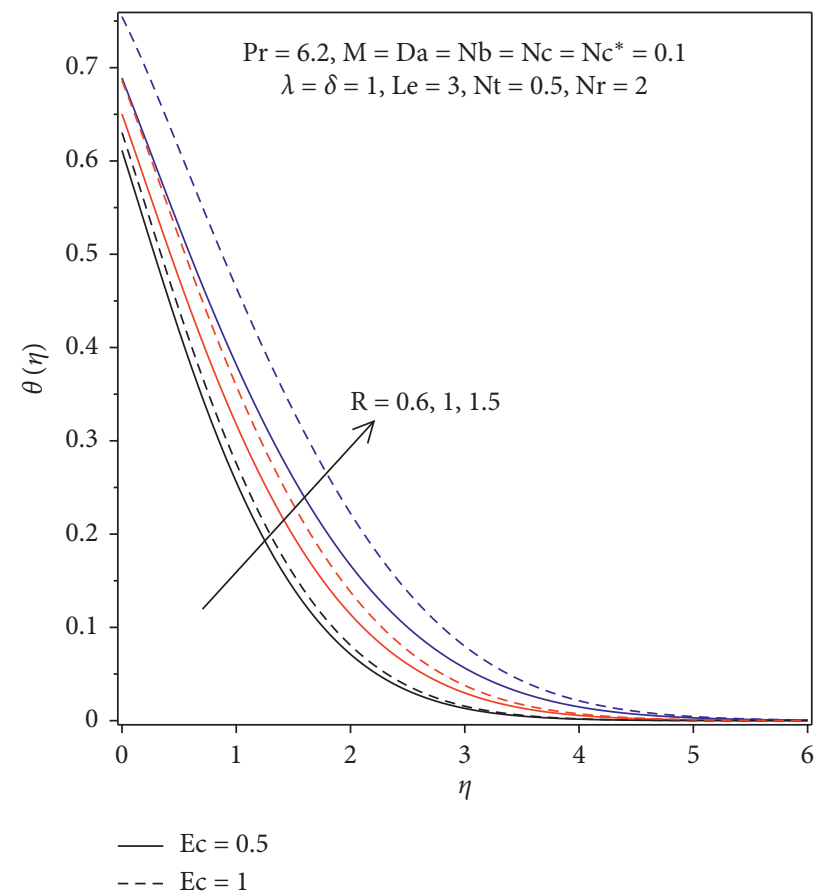

(b)

FIgURE 5: (a) Influence of $\mathrm{Nr}$ on the temperature profile. (b) Influence of $R$ and Ec on the temperature profile.

higher values of nanofluid buoyancy ratio parameter enhance the temperature of the fluid so that the thermal boundary layer thickness is increased. The effects of viscous dissipation on the dimensionless temperature profile for different values of thermal radiation parameters are depicted in Figure 5(b). In Figure 5(b), the intensification of viscous dissipation increases the temperature of the fluid. In viscous fluid flow, the viscosity of the fluid takes kinetic energy from the motion of the fluid and transforms it into internal energy that heats the fluid. Furthermore, it 
can be observed that radiation enhances the dimensionless temperature leading to the development of an ascending thermal boundary layer. Higher fluid temperature contributes to a reduction in heat transfer from the plate. Hence, it is expected that thermal radiation must be kept low to attain more cooling from the plate.

\subsection{Nanoparticle Volume Fraction Profiles with Parameter} Variations. The effects on nanoparticle volume fraction with emerging parameters are presented in Figure 6. From Figure 6(a), one can see that as the stretching/shrinking parameter increases, the nanoparticle volume fraction enhances up to the region of fluid $\eta=2$, followed by transition there, and it gets diminution with the same condition of $\lambda$, whereas an increase in magnetic field parameter resulted in decreasing nanoparticle volume fraction within a few layers of the fluid from the plate which is up to $\eta=2$ and just after the transition the effect is negligible.

The combined effects of the nanofluid parameters (Brownian and thermophoresis parameters) are shown in Figure 6(b). We can see that an increase in $N t$ shows decreasing effects on nanoparticle volume fraction within a few layers of the fluid from the plate, which is up to $\eta=1.6$. Afterwards, that is, in the region $\eta=1.6$ to $\eta=10$, the nanoparticle volume fraction undergoes the reverse impact due to increase in $N t$ followed by transition at $\eta=1.6$. This is because an increase in $\mathrm{Nt}$ indicates a strong thermophoretic force, leading to larger diffusion of nanoparticles from the hot plate to the ambient fluid. Furthermore, an increase in $\mathrm{Nb}$ shows increasing effects on nanoparticle volume fraction within a few layers of the fluid from the plate which is up to $\eta=1.6$ and follows a reverse trend just after the transition.

4.4. Skin Friction, Reduced Nusselt Number, and Reduced Sherwood Number Profiles. The skin friction variation with different parameters is shown in Figure 7. As presented in Figure $7(\mathrm{a})$, the skin friction is less for higher values of the radiation parameter. The effect of the magnetic field parameter is to increase skin friction due to viscous effects. Furthermore, the increase in the nanofluid buoyancy ratio parameter helps in decreasing skin friction. Figure 7 (b) characterizes the skin friction for increasing values of the convective parameter and Brownian motion parameters for the cases of permeable and impermeable sheets. As we can see from the graph, with an intensification in the convective parameter, the skin friction increases for the Brownian motion parameter and decreases for the suction/injection parameter.

Combined effects of nanofluid buoyancy parameter, magnetic field parameter, and stretching/shrinking parameter on reduced Nusselt number (a measure of heat transfer rate from the plate) is plotted in Figure 8(a). We can see that, with an increased magnetic field parameter, the reduced Nusselt number decreases, and, with an increase in nanofluid buoyancy parameter, the reduced Nusselt number increases. Also, the reduced Nusselt number is larger for the stretching sheet than for the shrinking sheet.
It is found in Figure 8(b) that a difference is appearing in the reduced Nusselt number for the convective parameter and the Brownian motion parameter for permeable and impermeable sheets. An increase in convective parameter increases the reduced Nusselt number, while opposite behavior is obtained on reduced Nusselt number for increasing values of the Brownian motion parameter for both permeable and impermeable sheets. As illustrated in this figure, suction assists the movement of heated fluid particles towards the wall and, subsequently, increases heat transfer rates compared to injection.

The effects of the magnetic field parameter and nanofluid buoyancy parameter in the presence of thermal radiation are depicted in Figure 9(a). The Sherwood number (mass transfer rate) increases with the magnetic field parameter and decreases with the nanofluid buoyancy parameter for the case of both occurrence and nonoccurrence of the thermal radiation parameter. Moreover, the Sherwood number has an inverse relationship with the thermal radiation parameter. Figure 9(b) shows the influence of the change of nanofluid parameters on the Sherwood number for different values of the buoyancy parameter. The Sherwood number upsurges with the thermophoresis parameter and falls with the Brownian motion parameter for all values of nanofluid buoyancy parameters.

The combined effects of buoyancy force, convective heating, viscous dissipation, and magnetic field parameters on skin friction and heat and mass transfer from a permeable stretching/shrinking sheet in a porous medium are investigated numerically, and obtained results are presented in Tables 1 and 2 .

4.5. Dual Solutions. Two real solutions exist within the valid range of shrinking parameter $\lambda<0$. These dual solutions exist in the range $\lambda_{c}<\lambda$ and no solution exists for $\lambda<\lambda_{c}$, where $\lambda_{c}$ is the critical value of shrinking parameter for which unique solution exists. It is well documented in the literature that only the upper solution branch for shrinking sheet is stable and physically realistic ([15-19]), while the other one diverges when subjected to disturbances. Therefore, only one valid solution exists within the range of shrinking parameter values.

As shown in Figure 10(a), the value of $s^{\prime \prime}(0)$ for the upper branch solution was observed to increase as $M$ increases. This shows that the increase in magnetic field parameters caused the wall shear stress to increase. Likewise, the value of $\left|\lambda_{c}\right|$ increased as $M$ increased. Furthermore, the effects of the porosity parameter on the critical value of the shrinking parameter $\left(\lambda_{c}\right)$ and wall shear stress is depicted in Figure 10(b). For the upper branch solution, the value of $s^{\prime \prime}(0)$ decreased as the porosity parameter $(\mathrm{Da})$ increased and the value of $\left|\lambda_{c}\right|$ decreased as Da increased.

As shown in Figures 10(a) and 10(b), the model exhibits a critical value for shrinking parameter values below which no solution exists. This implies that the sheet will stop shrinking when the critical value of the shrinking parameter is attained. This critical value also varies depending on the values of other emerging parameters. Moreover, it is 


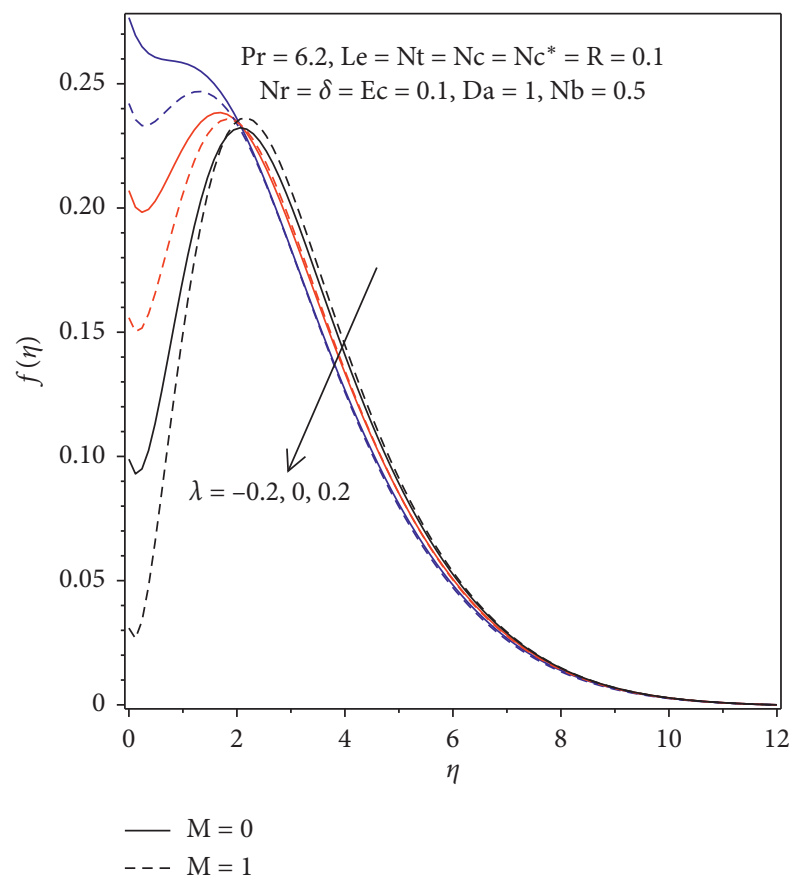

(a)

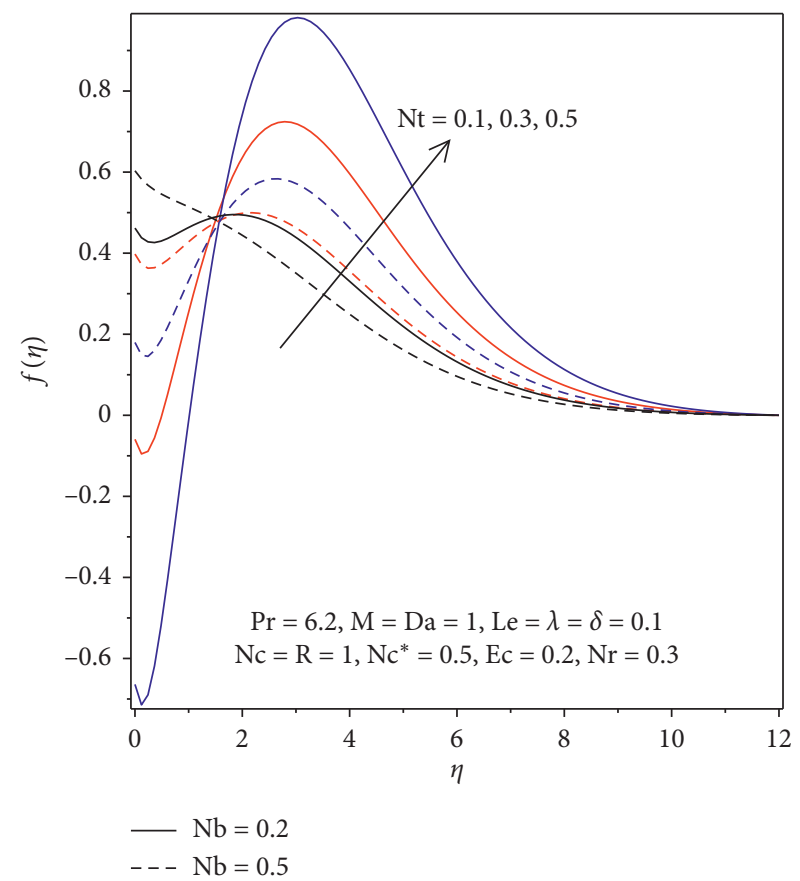

(b)

FIgURE 6: (a) Influence of $\lambda$ and $M$ on the concentration profile. (b) Influence of $\mathrm{Nt}$ and $\mathrm{Nb}$ on the concentration profile.

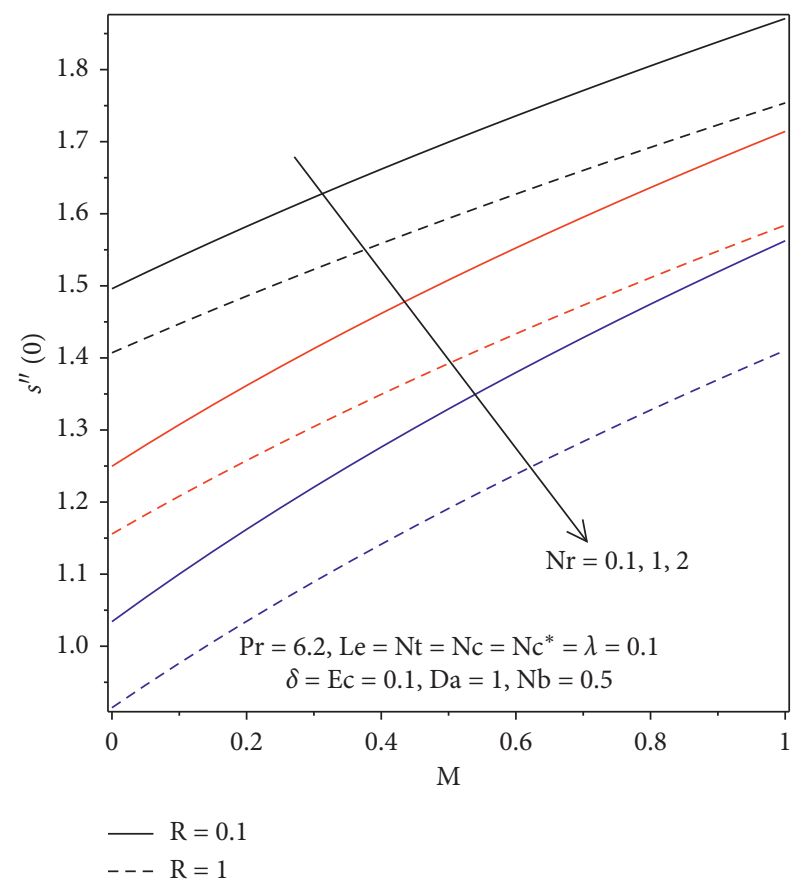

(a)

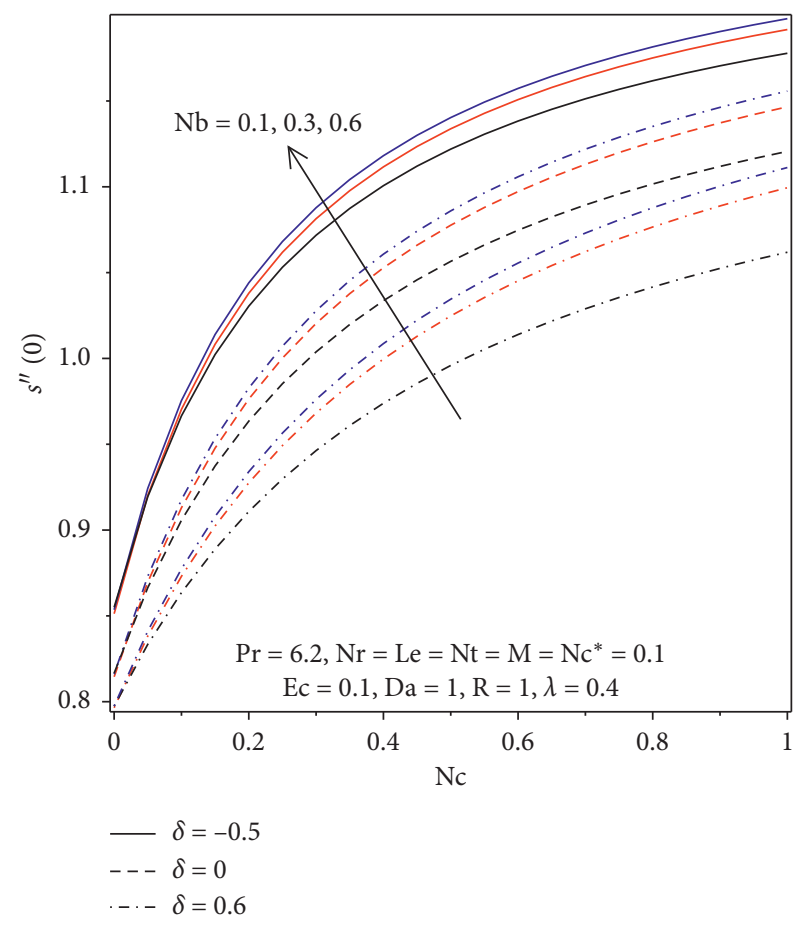

(b)

Figure 7: (a) Variation of skin friction with $M$ for different values of $\mathrm{Nr}$ and $R$. (b) Variation of skin friction with Nc for different values of $\mathrm{Nb}$ and $\delta$.

important to note that, for shrinking sheets, the convergence of the solution is limited with the critical parameter value $\left(\lambda_{c}\right)$. The solution only converges for a range of parameter values $\lambda_{c}<\lambda<0$ and the value of $\left(\lambda_{c}\right)$ depends on the values of other embedded thermophysical parameters in the governing model. The effects of those emerging parameters on 


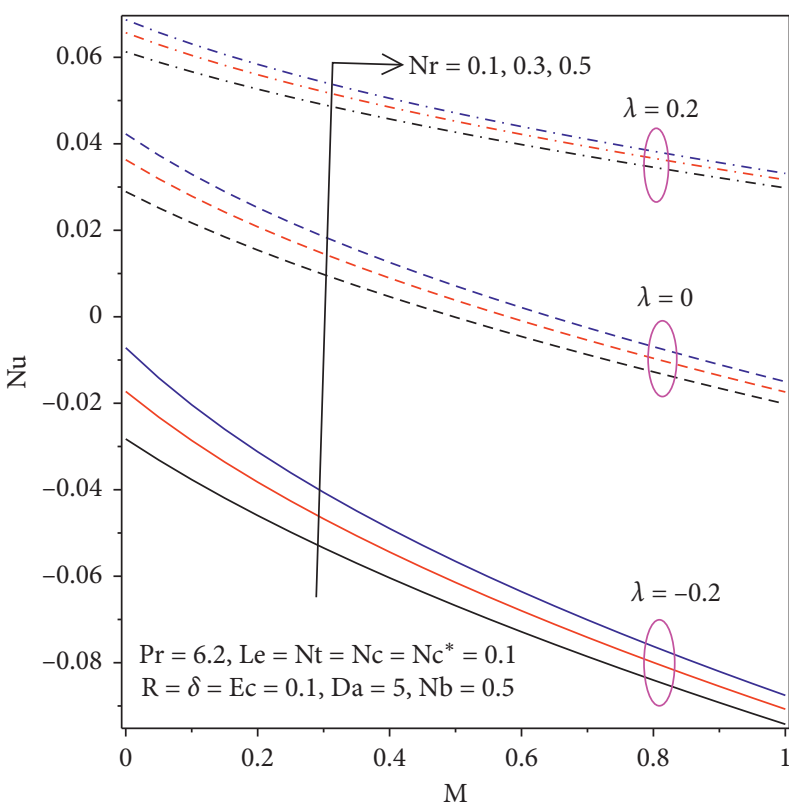

(a)

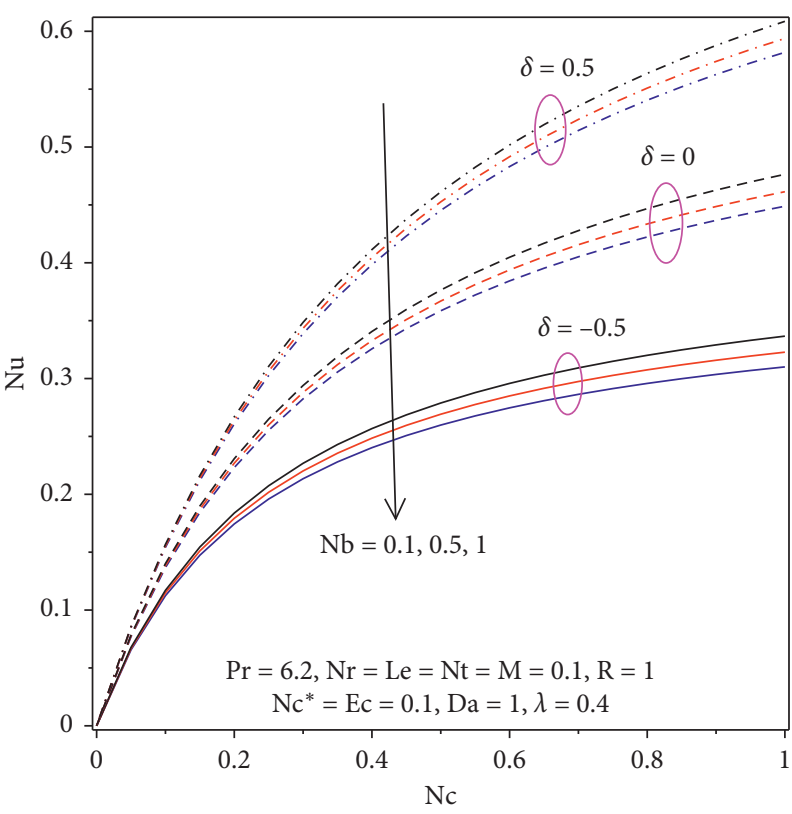

(b)

FiguRE 8: (a) Variation of reduced Nusselt number with $M$ for different values of $\mathrm{Nr}$ and $\lambda$. (b) Variation of reduced Nusselt number with Nc for different values of $\mathrm{Nb}$ and $\delta$.

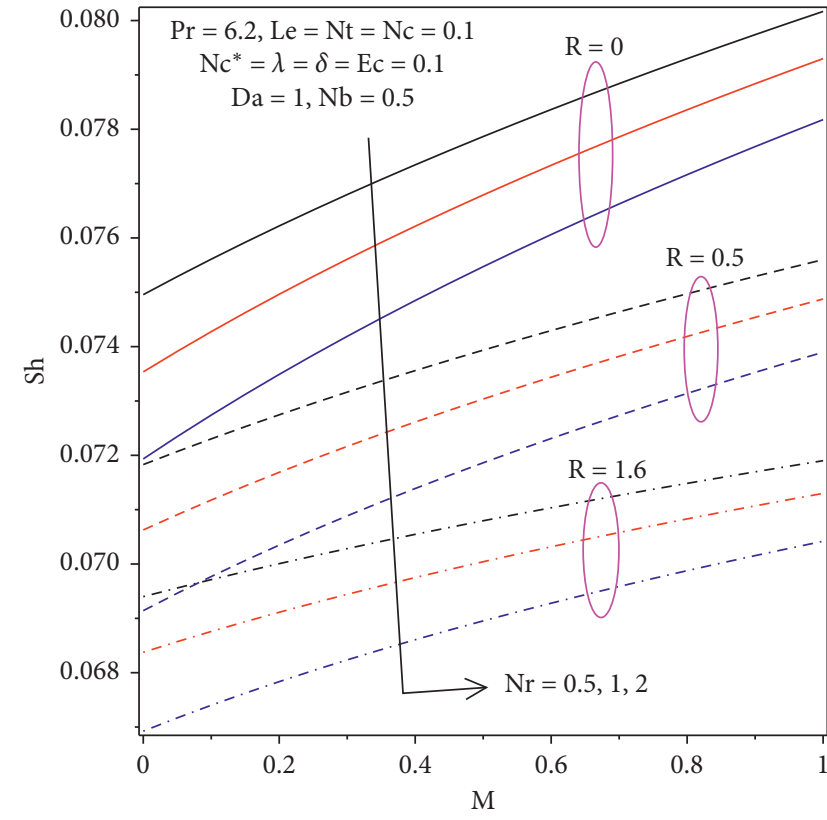

(a)

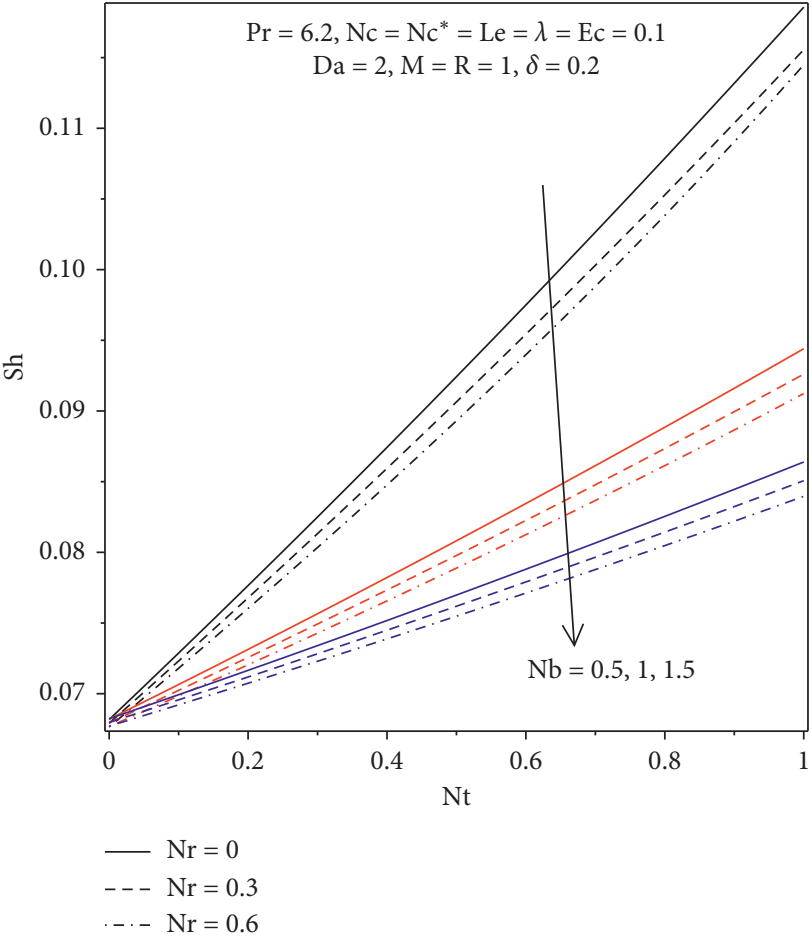

(b)

Figure 9: (a) Variation of Sh with $\mathrm{M}$ for different values of $\mathrm{Nr}$ and $\mathrm{R}$ (b) Variation of Sh with $\mathrm{Nt}$ for different values of $\mathrm{Nb}$ and $\mathrm{Nr}$. 
TABle 1: Computations showing the effects of buoyancy, magnetic, viscous dissipation, convective heating, and stretching/shrinking parameters on dimensionless temperature, shear stress, and heat and mass transfer rates when $\operatorname{Pr}=6.2, L e=3, N b=N t=0.3, N c^{*}=0.1, R=0.1, D a=1, \delta=0.1$.

\begin{tabular}{lccc}
\hline & $s^{\prime \prime}(0)$ & $-(1+4 / 3 R) \theta^{\prime}(0)$ & $-f^{\prime}(0)$ \\
\hline$\lambda$ & & $M=0.1, \mathrm{Ec}=0.1, \mathrm{Nc}=0.1, \mathrm{Nr}=1$ & 0.20268 \\
-0.3 & 3.72216 & -0.24874 & 0.12925 \\
0 & 1.96888 & -0.02765 & 0.09572 \\
0.5 & 0.73428 & 0.07519 & 0.1 \\
$\mathrm{Nr}$ & & $M=0.1, \mathrm{Ec}=0.1, \mathrm{Nc}=0.1, \lambda=-0.19478$ \\
0 & 2.20195 & -0.06593 & 0.14275 \\
0.3 & 2.25119 & -0.06742 & 0.14326 \\
0.5 & 2.28448 & -0.06847 & 0.14362 \\
1 & 2.36960 & -0.07134 & 0.14457 \\
$\mathrm{M}$ & & $\lambda=-0.1, \mathrm{Ec}=0.1, \mathrm{Nc}=0.1, \mathrm{Nr}=1$ & 0.14281 \\
0 & 2.31130 & -0.06600 & 0.15090 \\
0.5 & 2.57923 & -0.09083 & 0.15768 \\
1 & 2.80403 & -0.11221 & 0.22592 \\
10 & 5.03698 & -0.35215 & 1.58172 \\
$\mathrm{Ec}$ & & $M=0.1, \lambda=-0.1, \mathrm{Nc}=0.1, \mathrm{Nr}=1$ \\
0 & 1.25636 & 0.09333 & 0.08686 \\
0.5 & 2.36960 & -0.07134 & 0.14457 \\
$\mathrm{Nc}$ & & $M=0.1, \mathrm{Ec}=0.1, \lambda=-0.1, \mathrm{Nr}=1$ \\
0.1 & 2.36960 & -0.07134 & 0.14457 \\
1 & 2.12912 & -0.21083 & 0.14229 \\
10 & 2.04461 & -0.26926 & 1.50415 \\
& & & 0.14203 \\
\end{tabular}

TABle 2: Computations showing the effects of Brownian, suction/injection, thermophoresis, convective mass transfer, radiation, and the porous media parameters on dimensionless temperature, shear stress, and heat and mass transfer rates when $\operatorname{Pr}=6.2, \mathrm{Le}=0.1, \mathrm{Nr}=1, M=1, \mathrm{Ec}=\mathrm{Nc}=\lambda=0.1$.

\begin{tabular}{|c|c|c|c|c|}
\hline & $s^{\prime \prime}(0)$ & $-(1+(4 / 3) R) \theta^{\prime}(0)$ & $-f^{\prime}(0)$ & $\theta(0)$ \\
\hline$\delta$ & \multicolumn{4}{|c|}{$\mathrm{Nb}=\mathrm{Nt}=0.3, \mathrm{Nc}^{*}=0.1, R=0.1, \mathrm{Da}=0.1$} \\
\hline-0.5 & 10.21855 & -1.68520 & 1.23498 & 15.86937 \\
\hline 0 & 3.98653 & -0.16588 & 0.23885 & 2.46365 \\
\hline 0.5 & 3.64907 & -0.08893 & 0.17686 & 1.78465 \\
\hline $\mathrm{Nb}$ & \multicolumn{4}{|c|}{$\mathrm{Nt}=0.3, \delta=0.2, \mathrm{Nc}^{*}=0.1, R=0.1, \mathrm{Da}=0.1$} \\
\hline 0.3 & 3.82272 & -0.12910 & 0.20910 & 2.13908 \\
\hline 0.6 & 3.64867 & -0.12656 & 0.13818 & 2.11668 \\
\hline $\mathrm{Nt}$ & \multicolumn{4}{|c|}{$\mathrm{Nb}=0.3, \delta=0.2, \mathrm{Nc}^{*}=0.1, R=0.1, \mathrm{Da}=0.1$} \\
\hline 0.1 & 3.56831 & -0.11859 & 0.11336 & 2.04642 \\
\hline 0.3 & 3.82272 & -0.12910 & 0.20910 & 2.11668 \\
\hline $\mathrm{Nc}^{*}$ & \multicolumn{4}{|c|}{$\mathrm{Nb}=\mathrm{Nt}=0.3, \delta=0.2, R=0.1, \mathrm{Da}=0.1$} \\
\hline 0.1 & 3.82272 & -0.12910 & 0.20910 & 2.13908 \\
\hline 1 & 3.44429 & -0.14380 & 0.55853 & 2.26885 \\
\hline 10 & 3.33633 & -0.15366 & 0.67566 & 2.35579 \\
\hline $\mathrm{R}$ & \multicolumn{4}{|c|}{$\mathrm{Nb}=\mathrm{Nt}=0.3, \mathrm{Nc}^{*}=0.1, \delta=0.2, \mathrm{Da}=0.1$} \\
\hline 0 & 3.88340 & -0.12863 & 0.22256 & 2.28629 \\
\hline 1 & 3.53589 & -0.11228 & 0.14951 & 1.48121 \\
\hline 2 & 3.40631 & -0.07428 & 0.12457 & 1.20259 \\
\hline $\mathrm{Da}$ & \multicolumn{4}{|c|}{$\mathrm{Nb}=\mathrm{Nt}=0.3, \mathrm{Nc}^{*}=0.1, R=0.1, \delta=0.2$} \\
\hline 0.5 & 2.22301 & -0.02740 & 0.13541 & 1.24178 \\
\hline 2 & 1.62546 & 0.00655 & 0.10917 & 0.94220 \\
\hline 5 & 1.45769 & 0.01531 & 0.10201 & 0.86490 \\
\hline
\end{tabular}




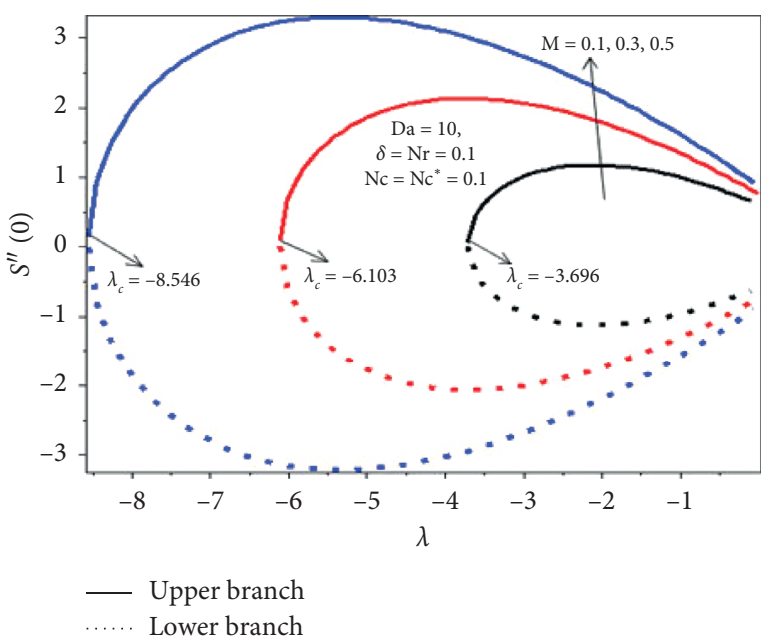

(a)

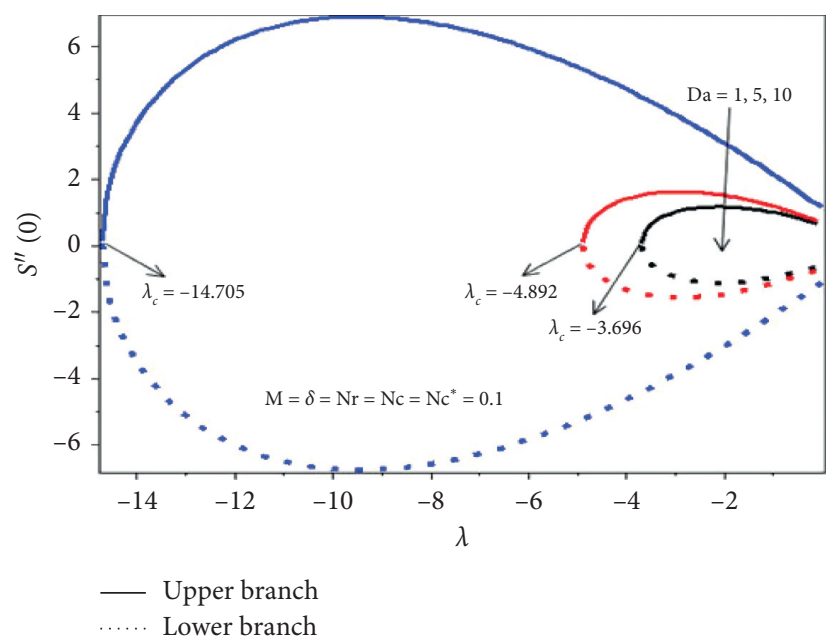

(b)

Figure 10: (a) Impact of $M$ on $\lambda_{c}$ and skin friction. (b) Impact of Da on $\lambda_{c}$ and skin friction.

TABLE 3: Computation showing critical shrinking parameter $\left(\lambda_{c}\right)$ when $\operatorname{Pr}=6.2$.

\begin{tabular}{|c|c|c|c|c|c|c|}
\hline $\mathbf{M}$ & Da & $\delta$ & $\mathrm{Nr}$ & $\mathrm{Nc}$ & $\mathrm{Nc}^{*}$ & $\lambda_{\mathrm{c}}$ \\
\hline 0.1 & 10 & 0.1 & 0.1 & 0.1 & 0.1 & -3.696 \\
\hline 0.3 & 10 & 0.1 & 0.1 & 0.1 & 0.1 & -6.103 \\
\hline 0.5 & 10 & 0.1 & 0.1 & 0.1 & 0.1 & -8.546 \\
\hline 0.1 & 5 & 0.1 & 0.1 & 0.1 & 0.1 & -4.892 \\
\hline 0.1 & 3 & 0.1 & 0.1 & 0.1 & 0.1 & -6.508 \\
\hline 0.1 & 1 & 0.1 & 0.1 & 0.1 & 0.1 & -14.705 \\
\hline 0.1 & 1 & 1.0 & 0.1 & 0.1 & 0.1 & -14.705 \\
\hline 0.1 & 1 & 0.1 & 0.5 & 0.1 & 0.1 & -14.676 \\
\hline 0.1 & 1 & 0.1 & 1.0 & 0.1 & 0.1 & -14.640 \\
\hline 0.1 & 1 & 0.1 & 0.1 & 0.5 & 0.1 & -14.893 \\
\hline 0.1 & 1 & 0.1 & 0.1 & 1.0 & 0.1 & -15.020 \\
\hline 0.1 & 1 & 0.1 & 0.1 & 1.0 & 0.5 & -14.686 \\
\hline 0.1 & 1 & 0.1 & 0.1 & 0.1 & 1.0 & -14.672 \\
\hline
\end{tabular}

TABLE 4: Comparison of values for skin friction and Nusselt number for different values of $\lambda$ and $N r$ with $M=1, \mathrm{Ec}=0.1, \mathrm{Nc}=1$ for the case of $\mathrm{Da}=\mathrm{Nc}^{*}=\infty, R=0, \delta=0$.

\begin{tabular}{|c|c|c|c|c|c|}
\hline \multirow{2}{*}{$\lambda$} & \multirow{2}{*}{$\mathrm{Nr}$} & \multicolumn{2}{|c|}{$S^{\prime \prime}(0)$} & \multicolumn{2}{|c|}{$\mathrm{Nu}$} \\
\hline & & Makinde et al. [15] & Present result & Makinde et al. [15] & Present result \\
\hline \multirow{3}{*}{-0.5} & 0 & 2.35476 & 2.3547617880 & 0.13379 & 0.13379056427 \\
\hline & 0.3 & 2.21267 & 2.2126690902 & 0.13425 & 0.13424984870 \\
\hline & 0.5 & 2.11589 & 2.1158915121 & 0.13409 & 0.13408574710 \\
\hline \multirow{3}{*}{0} & 0 & 1.73177 & 1.7317739823 & 0.25999 & 0.25999218413 \\
\hline & 0.3 & 1.60462 & 1.6046181450 & 0.26005 & 0.26005145639 \\
\hline & 0.5 & 1.51848 & 1.5184779144 & 0.25979 & 0.25978597088 \\
\hline \multirow{3}{*}{0.5} & 0 & 1.03430 & 1.0343034653 & 0.34415 & 0.34415369544 \\
\hline & 0.3 & 0.92053 & 0.92053122986 & 0.34357 & 0.34356698719 \\
\hline & 0.5 & 0.84380 & 0.84379568546 & 0.34297 & 0.34296800192 \\
\hline
\end{tabular}


critical value of the shrinking parameter $\left(\lambda_{c}\right)$ are presented in Table 3.

The convergence and validity of the solutions obtained are displayed in Table 4 . The special case of our numerical results was validated with the one already in the literature [15], and excellent agreement is achieved.

\section{Conclusion}

A problem of two-dimensional MHD stagnation point flow of an electrically conducting water-based nanofluid towards a permeable stretching/shrinking in a porous medium is solved numerically to investigate the combined effects of buoyancy ratio parameter, thermal radiation, porous medium, convective heating, viscous dissipation, magnetic field, and nanofluid parameters on the dimensionless velocity, temperature, rescaled nanoparticle volume fraction, skin friction, and heat and mass transfer rates from a permeable stretching/shrinking sheet. Similarity transformations technique is applied to transform the resulting model equations into nonlinear ordinary differential equations. The MAPLE software is used to generate the numerical solutions of the transformed equations with the boundary conditions. The conclusions are the following:

(i) The dimensionless velocity and temperature profiles decrease with porous medium parameter and mass suction/injection parameter, whereas an increase in radiation increases the temperature

(ii) The skin friction increases with both convective and Brownian motion parameters but decreases with increasing mass suction/injection, which is also lower for higher values of radiation parameter and shows intensification with magnetic field parameter

(iii) The reduced Nusselt numbers are higher for stretching sheets than for shrinking sheets and also decrease with magnetic field parameter and Brownian motion parameter but upsurge with nanofluid buoyancy ratio, heat convective parameter, and thermophoresis parameter

(iv) The Sherwood numbers increase with the magnetic field and thermophoresis parameter but decrease with nanofluid buoyancy ratio, thermal radiation parameter, and Brownian motion parameter

(v) Two real solutions exist within a specific range of shrinking parameter; however, only one of the two solutions is physically realistic and the other one diverges when subjected to disturbances

(vi) The value of $\left|\lambda_{c}\right|$ increases with increasing values of the magnetic field parameter $M$, whereas the value of $\left|\lambda_{c}\right|$ decreases with increasing value of the porosity parameter $\mathrm{Da}$

\section{Appendix}

\section{A. Derivation of the Problem}

The governing partial differential equations of the problem are

$$
\begin{aligned}
\frac{\partial u}{\partial x}+\frac{\partial v}{\partial y}= & 0 \\
u \frac{\partial u}{\partial x}+v \frac{\partial u}{\partial y}= & U_{\infty} \frac{\partial U_{\infty}}{\partial x}+\frac{\mu_{f}}{\rho_{f}} \frac{\partial^{2} u}{\partial y^{2}}-\frac{\sigma B_{0}^{2}}{\rho_{f}}\left(u-U_{\infty}\right)-\frac{\mu_{f}}{\rho_{f} K}\left(u-U_{\infty}\right) \\
& +\frac{1}{\rho_{f}}\left[\left(1-\phi_{\infty}\right) \rho_{f \infty} \beta g\left(T-T_{\infty}\right)-\left(\rho_{p}-\rho_{\infty}\right) g\left(\phi-\phi_{\infty}\right)\right] \\
u \frac{\partial T}{\partial x}+v \frac{\partial T}{\partial y}= & \alpha_{f}\left(1+\frac{16 \sigma^{*} T_{\infty}^{3}}{3 \mathrm{kk}^{*}}\right)\left(\frac{\partial^{2} T}{\partial y^{2}}\right)+\tau\left(D_{B} \frac{\partial \phi}{\partial y} \frac{\partial T}{\partial y}+\frac{D_{T}}{T_{\infty}}\left(\frac{\partial T}{\partial y}\right)^{2}\right) \\
& +\frac{\alpha_{f} \mu_{f}}{k}\left(\frac{\partial u}{\partial y}\right)^{2}+\left(\frac{\alpha_{f} \sigma B_{0}^{2}}{k}+\frac{\alpha_{f} \mu_{f}}{\mathrm{kK}}\right)\left(u-U_{\infty}\right)^{2} \\
u \frac{\partial \phi}{\partial x}+v \frac{\partial \phi}{\partial y}= & D_{B} \frac{\partial^{2} \phi}{\partial y^{2}}+\left(\frac{D_{T}}{T_{\infty}}\right)\left(\frac{\partial^{2} T}{\partial y^{2}}\right)
\end{aligned}
$$


with the boundary conditions,

$$
\begin{aligned}
y & =0: u=\mathrm{ax}, v=V_{0},-k \frac{\partial T}{\partial y}=h_{1}\left(T_{f}-T\right), \\
-D_{B} \frac{\partial \phi}{\partial y} & =h_{2}\left(\phi_{f}-\phi\right),
\end{aligned}
$$$$
y \longrightarrow \infty: u \longrightarrow U_{\infty}(x), T \longrightarrow T_{\infty}, \phi \longrightarrow \phi_{\infty},
$$

and then the following dimensionless variables are introduced:

$$
\eta=\frac{y}{x} \operatorname{Ra}_{x}^{1 / 4} \quad \psi=\alpha_{f} \operatorname{Ra}_{x}^{1 / 4} s(\eta) \quad \theta(\eta)=\frac{T-T_{\infty}}{T_{f}-T_{\infty}} \quad f(\eta)=\frac{\phi-\phi_{\infty}}{\phi_{w}-\phi_{\infty}} .
$$

Now, using the similarity transformation quantities, the governing equations are transformed to ordinary differential equations. We have

$$
\begin{aligned}
u & =\frac{\partial \psi}{\partial y}=\frac{\alpha_{f}}{x} \mathrm{Ra}_{x}^{1 / 4} s^{\prime}(\eta) \mathrm{Ra}_{x}^{1 / 4} \Longrightarrow u=\frac{\alpha_{f}}{x} \operatorname{Ra}_{x}^{1 / 2} s^{\prime}(\eta), \\
v & =-\frac{\partial \psi}{\partial x} \\
\Longrightarrow v & =-\alpha_{f}\left[\frac{-y}{4 x^{2}} \operatorname{Ra}_{x}^{1 / 2} s^{\prime}(\eta)+\frac{3}{4 x} \operatorname{Ra}_{x}^{1 / 4} s(\eta)\right], \\
T & =T_{\infty}+\left(T_{f}-T_{\infty}\right) \theta(\eta) \Longrightarrow \frac{\partial T}{\partial y}=\left(T_{f}-T_{\infty}\right) \theta^{\prime}(\eta) \frac{\mathrm{Ra}_{x}^{1 / 4}}{x}, \\
\Longrightarrow \frac{\partial^{2} T}{\partial y^{2}} & =\frac{\left(T_{f}-T_{\infty}\right)}{x^{2}} \theta^{\prime \prime}(\eta) \mathrm{Ra}_{x}^{1 / 2},
\end{aligned}
$$

and

and

$$
\begin{gathered}
\frac{\partial T}{\partial x}=\left(T_{f}-T_{\infty}\right) \theta^{\prime}(\eta) \frac{\mathrm{d} \eta}{\mathrm{d} x} \Longrightarrow-\frac{y}{4 x^{2}}\left(T_{f}-T_{\infty}\right) \theta^{\prime}(\eta) \mathrm{Ra}_{x}^{1 / 4}, \\
\phi=\phi_{\infty}+\left(\phi_{w}-\phi_{\infty}\right) f(\eta) \Longrightarrow \frac{\partial \phi}{\partial y}=\frac{\left(\phi_{w}-\phi_{\infty}\right)}{x} f^{\prime}(\eta) \mathrm{Ra}_{x}^{1 / 4} \\
\Longrightarrow \frac{\partial^{2} \phi}{\partial y^{2}}=\frac{\left(\phi_{w}-\phi_{\infty}\right)}{x^{2}} f^{\prime \prime}(\eta) \mathrm{Ra}_{x}^{1 / 2}
\end{gathered}
$$$$
\frac{\partial \phi}{\partial x}=\left(\phi_{w}-\phi_{\infty}\right) f^{\prime}(\eta) \frac{\mathrm{d} \eta}{\mathrm{d} x}=-\frac{y}{4 x^{2}}\left(\phi_{w}-\phi_{\infty}\right) f^{\prime}(\eta) \mathrm{Ra}_{x}^{1 / 4} .
$$

\section{A.1. The Continuity Equation}

$$
\begin{aligned}
\frac{\partial u}{\partial x}+\frac{\partial v}{\partial y} & =\frac{\partial}{\partial x}\left(\frac{\alpha_{f}}{x} \operatorname{Ra}_{x}^{1 / 2} s^{\prime}(\eta)\right)+\frac{\partial}{\partial y}\left(-\alpha_{f}\left[\frac{-y}{4 x^{2}} \mathrm{Ra}_{x}^{1 / 2} s^{\prime}(\eta)+\frac{3}{4 x} \mathrm{Ra}_{x}^{1 / 4} s(\eta)\right]\right) \\
& =0 \quad \text { (it shows that continuity equation is satisfied automatically). }
\end{aligned}
$$




\section{A.2. The Momentum Equation}

Deriving each expression separately based on equations (A.8) and (A.9), we obtain the following.

For the inertial term,

$$
\begin{aligned}
& u \frac{\partial u}{\partial x}+v \frac{\partial u}{\partial y} \\
& =\frac{-1}{x^{3}} \alpha_{f}^{2} \operatorname{Ra}_{x} s^{\prime}(\eta)^{2}+\frac{3}{2 x^{3}} \alpha_{f}^{2} \operatorname{Ra}_{x} s^{\prime}(\eta)^{2}-\frac{y}{4 x^{4}} \alpha_{f}^{2} \mathrm{Ra}_{x}^{5 / 4} s^{\prime} \\
& -\frac{3}{4 x^{3}} \alpha_{f}^{2} \operatorname{Ra}_{x} s(\eta) s^{\prime \prime}(\eta)+\frac{y}{4 x^{4}} \alpha_{f}^{2} \mathrm{Ra}_{x}^{5 / 4} s^{\prime}(\eta) s^{\prime \prime}(\eta) \\
& =\frac{1}{2 x^{3}} \alpha_{f}^{2} R a_{x}\left(s^{\prime}(\eta)\right)^{2}-\frac{3}{4 x^{3}} \alpha_{f}^{2} R a_{x} s^{\prime}(\eta) s^{\prime \prime}(\eta) \\
& \text { For the pressure term, } \\
& U_{\infty} \frac{\mathrm{d} U_{\infty}}{\mathrm{d} x}=\frac{1}{x} \alpha_{f} \operatorname{Ra}^{1 / 2}\left(-\frac{\alpha_{f}}{x^{2}} \operatorname{Ra}_{x}^{1 / 2}+\frac{3 \alpha_{f}}{2 x^{2}} \mathrm{Ra}_{x}^{1 / 2}\right) \\
& =\frac{1}{2 x^{3}} \alpha_{f}^{2} \operatorname{Ra}_{x} .
\end{aligned}
$$$$
=\frac{-1}{x^{3}} \alpha_{f}^{2} \operatorname{Ra}_{x} s^{\prime}(\eta)^{2}+\frac{3}{2 x^{3}} \alpha_{f}^{2} \operatorname{Ra}_{x} s^{\prime}(\eta)^{2}-\frac{y}{4 x^{4}} \alpha_{f}^{2} \operatorname{Ra}_{x}^{5 / 4} s^{\prime}(\eta) s^{\prime \prime}(\eta)
$$

For the viscous term,

$$
\begin{aligned}
\frac{\mu_{f}}{\rho_{f}} \frac{\partial^{2} u}{\partial y^{2}} & =\frac{\alpha_{f} \mu_{f}}{x^{2} \rho_{f}} \operatorname{Ra}_{x}^{3 / 4} s^{\prime \prime}(\eta) \frac{1}{x} \operatorname{Ra}_{x}^{1 / 4} \\
& =\frac{\alpha_{f} \mu_{f}}{x^{3} \rho_{f}} s^{\prime \prime}(\eta) \mathrm{Ra}_{x} .
\end{aligned}
$$

For the magnetic force term,

$$
\begin{aligned}
\frac{\sigma_{f} B_{0}^{2}}{\rho_{f}}\left(u-U_{\infty}\right) & =\frac{\sigma_{f} B_{0}^{2}}{\rho_{f}}\left(\frac{\alpha_{f}}{x} \operatorname{Ra}_{x}^{1 / 2} s^{\prime}(\eta)-\frac{\alpha_{f}}{x} \mathrm{Ra}_{x}^{1 / 2}\right) \\
& =\frac{\sigma_{f} B_{0}^{2}}{\rho_{f} x} \alpha_{f} \operatorname{Ra}_{x}^{1 / 2}\left(s^{\prime}(\eta)-1\right) .
\end{aligned}
$$

For porous medium term,

$$
\begin{aligned}
\frac{\mu_{f}}{\rho_{f} K}\left(u-U_{\infty}\right) & =\frac{\mu_{f}}{\rho_{f} K}\left(\frac{\alpha_{f}}{x} \operatorname{Ra}_{x}^{1 / 2} s^{\prime}(\eta)-\frac{\alpha_{f}}{x} \mathrm{Ra}_{x}^{1 / 2}\right) \\
& =\frac{\mu_{f}}{\rho_{f} K} \frac{\alpha_{f}}{x} \operatorname{Ra}_{x}^{1 / 2}\left(s^{\prime}(\eta)-1\right) .
\end{aligned}
$$

Substituting equations (A.17)-(A.21) in equation (A.2), we get

$$
\begin{aligned}
\frac{1}{2 x^{3}} \alpha_{f}^{2} \operatorname{Ra}_{x}\left(s^{\prime}(\eta)\right)^{2}-\frac{3}{4 x^{3}} \alpha_{f}^{2} \operatorname{Ra}_{x} s(\eta) s^{\prime \prime}(\eta)= & \frac{1}{2 x^{3}} \alpha_{f} \operatorname{Ra}_{x}-\frac{\alpha_{f} \mu_{f}}{x^{3} \rho_{f}} s^{\prime \prime \prime}(\eta) \mathrm{Ra}_{x} \\
& -\frac{\sigma_{f} B_{0}^{2}}{\rho_{f} x} \alpha_{f} \operatorname{Ra}_{x}^{1 / 2}\left(s^{\prime}(\eta)-1\right)-\frac{\mu_{f}}{\rho_{f} K} \frac{\alpha_{f}}{x} \operatorname{Ra}_{x}^{1 / 2}\left(s^{\prime}(\eta)-1\right) \\
& +\frac{1}{\rho_{f}}\left[\left(1-\phi_{\infty}\right) \rho_{f \infty} \beta g\left(T-T_{\infty}\right)-\left(\rho_{p}-\rho_{\infty}\right) g\left(\phi-\phi_{\infty}\right)\right]
\end{aligned}
$$

Dividing each term by $\mathrm{Ra}$, we get

$$
\begin{aligned}
\frac{1}{2 x^{3}} \alpha_{f}^{2}\left(s^{\prime}(\eta)\right)^{2}-\frac{3}{4 x^{3}} \alpha_{f}^{2} s(\eta) s^{\prime \prime}(\eta)= & \frac{\alpha_{f}^{2}}{2 x^{3}}-\frac{\alpha_{f} \mu_{f}}{x^{3} \rho_{f}} s^{\prime \prime \prime}(\eta)-\frac{\sigma_{f} B_{0}^{2}}{\rho_{f} x} \alpha_{f} \operatorname{Ra}_{x}^{-1 / 2}\left(s^{\prime}(\eta)-1\right) \\
& -\frac{\mu_{f}}{\rho_{f} K} \frac{\alpha_{f}}{x} \operatorname{Ra}_{x}^{1 /-2}\left(s^{\prime}(\eta)-1\right) \\
& +\frac{1}{\operatorname{Ra}_{x} \rho_{f}}\left[\left(1-\phi_{\infty}\right) \rho_{f \infty} \beta g\left(T-T_{\infty}\right)-\left(\rho_{p}-\rho_{\infty}\right) g\left(\phi-\phi_{\infty}\right)\right] .
\end{aligned}
$$


Multiplying each term by $x^{3} \rho_{f} / \mu_{f} \alpha_{f}$, we get

$$
\begin{aligned}
& s^{\prime \prime \prime}(\eta)-\frac{\alpha_{f} \rho_{f}}{2 \mu_{f}}\left(s^{\prime}(\eta)\right)^{2}+\frac{3 \alpha_{f} \rho_{f}}{4 \mu_{f}} s(\eta) s^{\prime \prime}(\eta)+\frac{1}{2 \operatorname{Pr}}-\frac{\sigma_{f} B_{0}^{2} x^{2}}{\mu_{f}} \operatorname{Ra}_{x}^{-1 / 2}\left(s^{\prime}(\eta)-1\right) \\
& -\frac{x^{2}}{K} \operatorname{Ra}_{x}^{1 /-2}\left(s^{\prime}(\eta)-1\right)+\frac{x^{3}}{\operatorname{Ra}_{x} \mu_{f} \alpha_{f}}\left[\left(1-\phi_{\infty}\right) \rho_{f \infty} \beta g\left(T-T_{\infty}\right)-\left(\rho_{p}-\rho_{\infty}\right) g\left(\phi-\phi_{\infty}\right)\right]=0 .
\end{aligned}
$$

After some simplifications and substitution of the parameters, we obtain

$$
\begin{aligned}
& s^{\prime \prime \prime}(\eta)-\left(M+\frac{1}{\mathrm{Da}}\right)\left(s^{\prime}(\eta)-1\right)-\frac{1}{2 \operatorname{Pr}}\left(s^{\prime}(\eta)\right)^{2}+\frac{3}{4 \operatorname{Pr}} s(\eta) s^{\prime \prime}(\eta)+\frac{1}{2 \operatorname{Pr}}+\frac{T-T_{\infty}}{T_{f}-T_{\infty}} \\
& -\frac{\left(\rho_{p}-\rho_{f}\right)\left(\phi_{w}-\phi_{\infty}\right)}{\rho_{f} \beta\left(1-\phi_{w}\right)\left(T_{f}-T_{\infty}\right)} \frac{\left(\phi-\phi_{\infty}\right)}{\left(\phi_{w}-\phi_{\infty}\right)}=0 .
\end{aligned}
$$

Finally, we obtain

$$
\begin{aligned}
& s^{\prime \prime \prime}(\eta)-\left(M+\frac{1}{\mathrm{Da}}\right)\left(s^{\prime}(\eta)-1\right)-\frac{1}{2 \operatorname{Pr}}\left(s^{\prime}(\eta)\right)^{2}+\frac{3}{4 \operatorname{Pr}} s(\eta) s^{\prime \prime}(\eta)+\frac{1}{2 \operatorname{Pr}}+\theta-\mathrm{Nrf}=0 \\
& s^{\prime \prime}-\left(M+\frac{1}{\mathrm{Da}}\right)\left(s^{\prime}-1\right)+\frac{1}{4 \operatorname{Pr}}\left[3 s s^{\prime \prime}-2 s^{2}+2\right]+\theta-\mathrm{Nrf}=0 .
\end{aligned}
$$

\section{A.3. The Energy Equation}

Substituting equations (A.8) and (A.10)-(A.13) into the energy equation (A.3), we obtain

$$
\begin{aligned}
& \frac{\alpha_{f}}{x} \mathrm{Ra}_{x}^{1 / 2} s^{\prime}(\eta)\left(-\frac{y}{4 x^{2}}\left(T_{f}-T_{\infty}\right) \theta^{\prime}(\eta) \mathrm{Ra}_{x}^{1 / 4}\right)+\left(\frac{y \alpha_{f}}{4 x^{2}} s^{\prime}(\eta) \mathrm{Ra}_{x}^{1 / 2}-\frac{3 \alpha_{f}}{4 x} s(\eta) \mathrm{Ra}_{x}^{1 / 4}\right) \\
& \frac{\left(T_{f}-T_{\infty}\right)}{x} \theta^{\prime}(\eta) \mathrm{Ra}_{x}^{1 / 4} \\
& =\left(1+\frac{16 \sigma^{*} T_{\infty}^{3}}{3 \mathrm{kk}^{*}}\right) \frac{\alpha_{f}}{x^{2}}\left(T_{f}-T_{\infty}\right) \theta^{\prime \prime}(\eta) \mathrm{Ra}_{x}^{1 / 2}+\tau\left(\frac{D_{T}}{T_{\infty}}\right)\left(\frac{\left(T_{f}-T_{\infty}\right)}{x} \theta^{\prime}(\eta) \mathrm{Ra}_{x}^{1 / 4}\right)^{2} \\
& +\tau\left[D_{B} \frac{\left(\phi_{w}-\phi_{\infty}\right)}{x} f^{\prime}(\eta) \operatorname{Ra}_{x}^{1 / 4} \frac{\left(T_{f}-T_{\infty}\right)}{x} \theta^{\prime}(\eta) \operatorname{Ra}_{x}^{1 / 4}\right] \frac{\alpha_{f} \mu_{f}}{k}\left(\frac{\alpha_{f}}{x^{2}} s^{\prime \prime}(\eta) \mathrm{Ra}_{x}^{3 / 4}\right)^{2} \\
& +\frac{\sigma B_{0}^{2} \alpha_{f}^{3}}{\mathrm{kx}^{2}} \operatorname{Ra}_{x}\left(s^{\prime}(\eta)-1\right)^{2}+\frac{\alpha_{f}^{3} \mu_{f}}{\mathrm{kKx}^{2}} \operatorname{Ra}_{x}\left(s^{\prime}(\eta)-1\right)^{2},
\end{aligned}
$$




$$
\begin{aligned}
-\frac{3 \alpha_{f}}{4 x^{2}}\left(T_{f}-T_{\infty}\right) s(\eta) \theta^{\prime}(\eta) \mathrm{Ra}_{x}^{1 / 2}= & \left(1+\frac{4}{3} \mathrm{Nr}\right) \frac{\alpha_{f}}{x^{2}}\left(T_{f}-T_{\infty}\right) \theta^{\prime \prime}(\eta) \mathrm{Ra}_{x}^{1 / 2} \\
& +\tau\left[D_{B} \frac{\left(\phi_{w}-\phi_{\infty}\right)\left(T_{f}-T_{\infty}\right)}{x^{2}} f^{\prime}(\eta) \theta^{\prime}(\eta) \mathrm{Ra}_{x}^{1 / 2}\right]+\tau\left(\frac{D_{T}}{T_{\infty}}\right)\left(\frac{\left(T_{f}-T_{\infty}\right)}{x} \theta^{\prime}(\eta) \mathrm{Ra}_{x}^{1 / 4}\right)^{2} \\
& +\frac{\alpha_{f} \mu_{f}}{k}\left(\frac{\alpha_{f}}{x^{2}} s^{\prime \prime}(\eta) \mathrm{Ra}_{x}^{3 / 4}\right)^{2}+\frac{\sigma B_{0}^{2} \alpha_{f}^{3}}{\mathrm{kx}^{2}} \operatorname{Ra}_{x}\left(s^{\prime}(\eta)-1\right)^{2}+\frac{\alpha_{f}^{3} \mu_{f}}{\mathrm{kKx}^{2}} \operatorname{Ra}_{x}\left(s^{\prime}(\eta)-1\right)^{2} .
\end{aligned}
$$

Multiplying each term by $x^{2} / \alpha_{f}\left(T_{f}-T_{\infty}\right) \mathrm{Ra}_{x}^{1 / 2}$, we obtain

$$
\begin{aligned}
& \left(1+\frac{4}{3} \mathrm{Nr}\right) \theta^{\prime \prime}(\eta)+\frac{3}{4} s(\eta) \theta^{\prime}(\eta)+\frac{\tau D_{B}\left(\phi_{w}-\phi_{\infty}\right)}{\alpha_{f}} f^{\prime}(\eta) \theta^{\prime}(\eta)+\frac{\tau D_{T}\left(T_{f}-T_{\infty}\right)}{T_{\infty} \alpha_{f}} \theta^{\prime}(\eta)^{2} \\
& +\frac{\alpha_{f}^{2} \mu_{f}}{x^{2} k\left(T_{f}-T_{\infty}\right)} \operatorname{Ra}_{x} s^{\prime \prime}(\eta)^{2}+\frac{\sigma B_{0}^{2}(x) \alpha_{f}^{2}}{k\left(T_{f}-T_{\infty}\right)} \operatorname{Ra}_{x}^{1 / 2}\left(s^{\prime}(\eta)-1\right)^{2} \\
& +\frac{\alpha_{f}^{2} \mu_{f}}{\operatorname{kK}\left(T_{f}-T_{\infty}\right)} \operatorname{Ra}_{x}^{1 / 2}\left(s^{\prime}(\eta)-1\right)^{2}=0
\end{aligned}
$$

and, after some simplification, we obtain

$$
\begin{aligned}
\theta^{\prime \prime}(\eta)+\frac{3}{4} s(\eta) \theta^{\prime}(\eta)+\mathrm{Nbf}^{\prime}(\eta) \theta^{\prime}(\eta)+\mathrm{Nt} \theta^{\prime}(\eta)^{2}+\operatorname{EcPr}^{\prime \prime}(\eta)^{2}+\operatorname{EcPr} M\left(s^{\prime}(\eta)-1\right)^{2} & =0 \\
\theta^{\prime \prime}+\frac{3}{4} s \theta^{\prime}+\operatorname{EcPr}\left[s^{\prime \prime 2}+M\left(s^{\prime}-1\right)^{2}\right]+\mathrm{Nbf}^{\prime} \theta^{\prime}+\mathrm{Nt} \theta^{\prime 2} & =0
\end{aligned}
$$

\section{A.4. The Concentration Equation}

Substituting equations (A.10), (A.11), (A.13), and (14) in (A.4), we obtain

$$
\begin{aligned}
\frac{\alpha_{f}}{x} & \operatorname{Ra}_{x}^{1 / 2} s^{\prime}(\eta)\left(-\frac{y}{4 x^{2}}\left(\phi_{w}-\phi_{\infty}\right) f^{\prime}(\eta) \mathrm{Ra}_{x}^{1 / 4}\right)+\frac{\left(\phi_{w}-\phi_{\infty}\right)}{x} f^{\prime}(\eta) \mathrm{Ra}_{x}^{1 / 4}, \\
{\left[\frac{y}{4 x^{2}} \alpha_{f} s^{\prime}(\eta) \mathrm{Ra}_{x}^{1 / 2}-\frac{3}{4 x} \alpha_{f} s(\eta) \mathrm{Ra}_{x}^{1 / 4}\right]=} & D_{B} \frac{\left(\phi_{w}-\phi_{\infty}\right)}{x^{2}} f^{\prime \prime}(\eta) \mathrm{Ra}_{x}^{1 / 2} \\
& +\left(\frac{D_{T}}{T_{\infty}}\right) \frac{\left(T_{f}-T_{\infty}\right)}{x^{2}} \theta^{\prime \prime}(\eta) \mathrm{Ra}_{x}^{1 / 2}, \\
& -\frac{y \alpha_{f}}{4 x^{3}}\left(\phi_{w}-\phi_{\infty}\right) \operatorname{Ra}_{x}^{3 / 4} s^{\prime}(\eta) f^{\prime}(\eta)+\frac{y \alpha_{f}}{4 x^{3}}\left(\phi_{w}-\phi_{\infty}\right) \operatorname{Ra}_{x}^{3 / 4} s^{\prime}(\eta) f^{\prime}(\eta) \\
-\frac{3}{4 x^{2}} \alpha_{f} f^{\prime}(\eta) s(\eta) \operatorname{Ra}_{x}^{1 / 2}= & D_{B} \frac{\left(\phi_{w}-\phi_{\infty}\right)}{x^{2}} f^{\prime \prime}(\eta) \operatorname{Ra}_{x}^{1 / 2}+\left(\frac{D_{T}}{T_{\infty}}\right) \frac{\left(T_{f}-T_{\infty}\right)}{x^{2}} \theta^{\prime}(\eta) \operatorname{Ra}_{x}^{1 / 2} .
\end{aligned}
$$


Now, multiplying each term by $x^{2} / D_{B}\left(\phi_{w}-\phi_{\infty}\right) \mathrm{Ra}_{x}^{1 / 2}$, we obtain

$$
\begin{aligned}
& \left(\frac{x^{2}}{D_{B}\left(\phi_{w}-\phi_{\infty}\right) \mathrm{Ra}_{x}^{1 / 2}}\right) \frac{-3}{4 x^{2}} \alpha_{f} f^{\prime}(\eta) s(\eta) \mathrm{Ra}_{x}^{1 / 2} \\
& =D_{B} \frac{\left(\phi_{w}-\phi_{\infty}\right)}{x^{2}} f^{\prime \prime}(\eta) \mathrm{Ra}_{x}^{1 / 2}\left(\frac{x^{2}}{D_{B}\left(\phi_{w}-\phi_{\infty}\right) \mathrm{Ra}_{x}^{1 / 2}}\right)+\left(\frac{x^{2}}{D_{B}\left(\phi_{w}-\phi_{\infty}\right) \mathrm{Ra}_{x}^{1 / 2}}\right) \\
& \left(\frac{D_{T}}{T_{\infty}}\right) \frac{\left(T_{f}-T_{\infty}\right)}{x^{2}} \theta^{\prime \prime}(\eta) \mathrm{Ra}_{x}^{1 / 2}, \\
& f^{\prime \prime}(\eta)+\frac{D_{T}\left(T_{f}-T_{\infty}\right)}{T_{\infty} D_{B}\left(\phi_{w}-\phi_{\infty}\right)} \theta^{\prime \prime}(\eta)+\frac{3}{4} \frac{\alpha_{f}}{D_{B}} f^{\prime}(\eta) s(\eta)=0, \\
& f^{\prime \prime}+\frac{\mathrm{Nt}}{\mathrm{Nb}} \theta^{\prime \prime}+\frac{3}{4} \operatorname{Lef}^{\prime} s=0 .
\end{aligned}
$$

The boundary conditions will be the following.

As $\eta \longrightarrow 0$,

$$
\begin{aligned}
& u(x, 0)=\operatorname{ax}=\frac{\alpha_{f}}{x} \operatorname{Ra}_{x}^{1 / 2} s^{\prime}(0) \Longrightarrow s^{\prime}(0)=\frac{\mathrm{ax}^{2}}{\alpha_{f} \sqrt{\mathrm{Ra}_{x}}}=\lambda, \\
& v(x, 0)=0=-\alpha_{f}\left[-\frac{y}{4 x^{2}} \mathrm{Ra}_{x}^{1 / 2} s^{\prime}(0)+\frac{3}{4 x} \mathrm{Ra}_{x}^{1 / 4} s(0)\right] \Longrightarrow s(0)=0 .
\end{aligned}
$$

Substituting equation (A.10) in the convective boundary equation (A.5), we obtain

$\theta^{\prime}(0)=\frac{x}{-k\left(T_{f}-T_{\infty}\right) \mathrm{Ra}_{x}^{1 / 4}} h_{f}(x)\left(T_{f}-T\right) \Longrightarrow \theta^{\prime}(0)=-\mathrm{Nc}(1-\theta(0))$.
Substituting equation (A.13) in the convective boundary equation (A.5), we obtain

$$
\theta^{\prime}(0)=\frac{x}{-D_{B}\left(\phi_{f}-\phi_{\infty}\right) \mathrm{Ra}_{x}^{1 / 4}} h_{f}(x)\left(\phi_{f}-\phi\right) \Longrightarrow f^{\prime}(0)=-\mathrm{Nc}^{*}(1-f(0)) .
$$

As $\eta \longrightarrow \infty$,

$$
\begin{aligned}
u(x, \infty) & =U_{\infty}(x)=\frac{\alpha_{f}}{x} \mathrm{Ra}_{x}^{1 / 2} s^{\prime}(\infty) \Longrightarrow \frac{\alpha_{f}}{x} \mathrm{Ra}_{x}^{1 / 2}=\frac{\alpha_{f}}{x} \mathrm{Ra}_{x}^{1 / 2} s^{\prime}(\infty) \Longrightarrow s^{\prime}(\infty)=1, \\
\theta(\infty) & =\frac{T(x, \infty)-T_{\infty}}{T_{f}-T_{\infty}}=\frac{T_{\infty}-T_{\infty}}{T_{f}-T_{\infty}} \Longrightarrow \theta(\infty)=0 \\
f(\infty) & =\frac{\phi(x, \infty)-\phi_{\infty}}{\phi_{w}-\phi_{\infty}}=\frac{\phi_{\infty}-\phi_{\infty}}{\phi_{w}-\phi_{\infty}} \Longrightarrow f(\infty)=0 .
\end{aligned}
$$




\section{Abbreviations}

a: Constant

$B_{0}$ : Magnitude of magnetic field

$D_{B}$ : Brownian diffusion coefficient $\left(\mathrm{m}^{-2} \mathrm{~s}^{-1}\right)$

$D_{T}$ : Thermophoresis diffusion coefficient $\left(\mathrm{m}^{-2} \mathrm{~s}^{-1}\right)$

$f$ : Dimensionless nanoparticle volume fraction

$k$ : $\quad$ Effective thermal conductivity of nanofluid $\left(\mathrm{Wm}^{-2} \mathrm{~K}^{-1}\right)$

$h_{f}$ : Heat transfer coefficient $\left(\mathrm{Wm}^{-2} \mathrm{~K}^{-1}\right)$

$k_{p}$ : Thermal conductivity of nanoparticles $\left(\mathrm{Wm}^{-2} \mathrm{~K}^{-1}\right)$

$k_{f}$ : Thermal conductivity of base fluid $\left(\mathrm{Wm}^{-2} \mathrm{~K}^{-1}\right)$

$\mathrm{Nb}$ : Brownian motion parameter

Nr: Reduced Nusselt number

Sh: Reduced local Sherwood number

$K$ : $\quad$ Porous medium permeability $\left(\mathrm{m}^{2}\right)$

$k^{*}$ : Mean absorption coefficient

$q_{w}^{\prime \prime} \quad$ Wall heat flux $\left(\mathrm{Wm}^{-2}\right)$

$q_{m}^{\prime \prime} \quad$ Mass flux $\left(\mathrm{kgs}^{-1} \mathrm{~m}^{-2}\right)$.

\section{Greek Symbols}

$\beta$ : $\quad$ Volumetric thermal expansion coefficient $\left(\mathrm{K}^{-1}\right)$

$\alpha_{f}$ : Thermal diffusivity of fluid $\left(\mathrm{m}^{2} \mathrm{~s}^{-1}\right)$

$\lambda$ : $\quad$ Stretching/shrinking parameter

$\phi: \quad$ Nanoparticle volume fraction $\left(\mathrm{kgm}^{-3}\right)$

$\phi_{w}: \quad$ Nanoparticle volume fraction at the wall $\left(\mathrm{kgm}^{-3}\right)$

$\eta$ : $\quad$ Similarity variable

$\phi_{\infty}$ : Ambient nanoparticle volume fraction $\left(\mathrm{kgm}^{-3}\right)$

$\mu$ : $\quad$ Absolute viscosity $\left(\mathrm{kgm}^{-1} \mathrm{~s}^{-1}\right)$

$v$ : $\quad$ Kinematic viscosity of the fluid $\left(\mathrm{m}^{2} \mathrm{~s}^{-1}\right)$

$\rho_{f}: \quad$ Fluid density $\left(\mathrm{kgm}^{-3}\right)$

$\rho_{p}: \quad$ Nanoparticle mass density $\left(\mathrm{kgm}^{-3}\right)$

$(\rho c)_{f}$ : Heat capacity of the fluid $\left(\mathrm{JK}^{-1} \mathrm{~kg}^{-1}\right)$

$\mathrm{Ra}_{\mathrm{x}}$ : Local Rayleigh number

Pr: Prandtl number

Le: Lewis number

Nc: Convective parameter

$T: \quad$ Temperature (K)

$T_{f}$ : Local fluid temperature $(\mathrm{K})$

$T_{\infty}$ : Ambient temperature (K)

$s: \quad$ Dimensionless stream function

$g$ : Acceleration due to gravity $\left(\mathrm{ms}^{-2} \mathrm{~ms}^{-2}\right)$

$U_{\infty}:$ External velocity $\left(\mathrm{ms}^{-1}\right)$

$u, v$ : Velocity components along $x$-axis and $y$-axis $\left(\mathrm{ms}^{-1}\right)$

$x, y$ : Coordinate along the plate and normal to it $(\mathrm{m})$

$(\rho c)_{p}$ : Heat capacity of the nanoparticle $\left(\mathrm{JK}^{-1} \mathrm{~g}^{-1}\right)$

$\theta$ : Dimensionless temperature

$\psi$ : $\quad$ Stream function

$\sigma: \quad$ Electrical conductivity $\left(\mathrm{Sm}^{-1}\right)$.

\section{Data Availability}

No data were used to support this study.

\section{Conflicts of Interest}

The authors declare that there are no conflicts of interest.

\section{Acknowledgments}

The authors gratefully acknowledge the financial support by Adama Science and Technology University under grant no. ASTU/SP-R/013/19.

\section{References}

[1] Y. Menni, A. J. Chamkha, and A. Azzi, "Nanofluid transport in porous media: a review," Special Topics \& Reviews in Porous Media: An International Journal, vol. 10, no. 1, pp. 49-64, 2019.

[2] S. Kakaç and A. Pramuanjaroenkij, "Review of convective heat transfer enhancement with nanofluids," International Journal of Heat and Mass Transfer, vol. 52, no. 13-14, pp. 3187-3196, 2009.

[3] S. U. S. Choi, "Enhancing thermal conductivity of fluids with nano-particles," ASME, FED, vol. 231, pp. 99-105, 1995.

[4] M. H. Toosi and M. Siavashi, "Two-phase mixture numerical simulation of natural convection of nanofluid flow in a cavity partially filled with porous media to enhance heat transfer," Journal of Molecular Liquids, vol. 238, pp. 553-569, 2017.

[5] R. A. Mahdi, H. A. Mohammed, K. M. Munisamy, and N. H. Saeid, "Review of convection heat transfer and fluid flow in porous media with nanofluid," Renewable and Sustainable Energy Reviews, vol. 41, pp. 715-734, 2015.

[6] A. Kasaeian, R. Daneshazarian, O. Mahian et al., "Nanofluid flow and heat transfer in porous media: a review of the latest developments," International Journal of Heat and Mass Transfer, vol. 107, pp. 778-791, 2017.

[7] A. J. Chamkha and M. A. Ismael, "Conjugate heat transfer in a porous cavity filled with nanofluids and heated by a triangular thick wall," International Journal of Thermal Sciences, vol. 67, pp. 135-151, 2013.

[8] R. A. Mahdi, H. a. Mohammed, and K. M. Munisamy, "Improvement of convection heat transfer by using porous media and nanofluid: review," International Journal of Science and Research, vol. 2, 2013.

[9] Q. Sun and I. Pop, "Free convection in a tilted triangle porous cavity filled with $\mathrm{Cu}$-water nanofluid with flush mounted heater on the wall," International Journal of Numerical Methods for Heat \& Fluid Flow, vol. 24, pp. 2-20, 2014.

[10] S. Yekani Motlagh, S. Taghizadeh, and H. Soltanipour, "Natural convection heat transfer in an inclined square enclosure filled with a porous medium saturated by nanofluid using Buongiorno's mathematical model," Advanced Powder Technology, vol. 27, no. 6, pp. 2526-2540, 2016.

[11] M. Sheikholeslami and D. D. Ganji, "Nanofluid flow and heat transfer in porous media," Applications of Nanofluid for Heat Transfer Enhancement, vol. 107, pp. 475-526, 2017.

[12] Y. Y. Lok, A. Ishak, and I. Pop, "MHD stagnation-point flow towards a shrinking sheet," International Journal of Numerical Methods for Heat \& Fluid Flow, vol. 21, no. 1, pp. 61-72, 2011.

[13] S. K. Soid, A. Ishak, and I. Pop, "MHD stagnation-point flow over a stretching/shrinking sheet in a micropolar fluid with a slip boundary," Sains Malaysiana, vol. 47, no. 11, pp. 2907-2916, 2018.

[14] W. Ibrahim, B. Shankar, and M. M. Nandeppanavar, "MHD stagnation point flow and heat transfer due to nanofluid towards a stretching sheet," International Journal of Heat and Mass Transfer, vol. 56, no. 1-2, pp. 1-9, 2013. 
[15] O. D. Makinde, W. A. Khan, and Z. H. Khan, "Buoyancy effects on MHD stagnation point flow and heat transfer of a nanofluid past a convectively heated stretching/shrinking sheet," International Journal of Heat and Mass Transfer, vol. 62, pp. 526-533, 2013.

[16] K. Jafar, A. Ishak, and R. Nazar, "MHD stagnation-point flow over a nonlinearly stretching/shrinking sheet," Journal of Aerospace Engineering, vol. 26, no. 4, pp. 829-834, 2013.

[17] R. Sharma, A. Ishak, and I. Pop, "Stability analysis of magnetohydrodynamic stagnation-point flow toward a stretching/ shrinking sheet," Computers \& Fluids, vol. 102, pp. 94-98, 2014.

[18] N. Freidoonimehr, M. M. Rashidi, and B. Jalilpour, "MHD stagnation-point flow past a stretching/shrinking sheet in the presence of heat generation/absorption and chemical reaction effects," Journal of the Brazilian Society of Mechanical Sciences and Engineering, vol. 38, no. 7, pp. 1999-2008, 2016.

[19] M. M. Bhatti, T. Abbas, and M. M. Rashidi, "A new numerical simulation of MHD stagnation-point flow over a permeable stretching/shrinking sheet in porous media with heat transfer," Iranian Journal of Science and Technology, Transactions A: Science, vol. 41, no. 3, pp. 779-785, 2017.

[20] O. Makinde, W. Khan, and Z. Khan, "Stagnation point flow of MHD chemically reacting nanofluid over a stretching convective surface with slip and radiative heat," Proceedings of the Institution of Mechanical Engineers, Part E: Journal of Process Mechanical Engineering, vol. 231, no. 4, pp. 695-703, 2017.

[21] K. U. Rehman, A. A. Khan, M. Y. Malik, and O. D. Makinde, "Thermophysical aspects of stagnation point magnetonanofluid flow yields by an inclined stretching cylindrical surface: a non-Newtonian fluid model," Journal of the Brazilian Society of Mechanical Sciences and Engineering, vol. 39, no. 9, pp. 3669-3682, 2017.

[22] R. Jusoh and R. Nazar, "MHD stagnation point flow and heat transfer of a nanofluid over a permeable nonlinear stretching/ shrinking sheet with viscous dissipation effect," AIP Conference Proceedings, vol. 1940, 2018.

[23] P. R. Sharma, S. Sinha, R. S. Yadav, and A. N. Filippov, "MHD mixed convective stagnation point flow along a vertical stretching sheet with heat source/sink," International Journal of Heat and Mass Transfer, vol. 117, pp. 780-786, 2018.

[24] S. Baag, S. R. Mishra, and B. Nayak, "Buoyancy effects on free convective MHD flow in the presence of heat source/sink," Modelling, Measurement and Control B, vol. 86, pp. 14-32, 2017.

[25] A. A. Abdullah, F. S. Ibrahim, and A. J. Chamkha, "Nonsimilar solution of unsteady mixed convection flow near the stagnation point of a heated vertical plate in a porous medium saturated with a nanofluid," Journal of Porous Media, vol. 21, no. 4, pp. 363-388, 2018.

[26] A. Jamaludin, R. Nazar, and I. Pop, "Mixed convection stagnation-point flow of a nanofluid past a permeable stretching/shrinking sheet in the presence of thermal radiation and heat source/sink," Energies, vol. 12, no. 5, p. 788, 2019.

[27] B. Jamil, M. S. Anwar, A. Rasheed, and M. Irfan, "MHD Maxwell flow modeled by fractional derivatives with chemical reaction and thermal radiation," Chinese Journal of Physics, vol. 67 , pp. 512-533, 2020.

[28] V. R. Prasad, N. Bhaskar Reddy, R. Muthucumaraswamy, and B. Vasu, "Transient radiative hydromagnetic free convection flow past an impulsively started vertical plate with uniform heat and mass flu," Journal of Applied Fluid Mechanics, vol. 4, pp. 59-68, 2006.
[29] O. D. Makinde, "Heat and mass transfer by MHD mixed convection stagnation point flow toward a vertical plate embedded in a highly porous medium with radiation and internal heat generation," Meccanica, vol. 47, no. 5, pp. 1173-1184, 2012.

[30] M. H. Mat Yasin, A. Ishak, I. Pop et al., "MHD heat and mass transfer flow over a permeable stretching/shrinking sheet with radiation effect," Journal of Magnetism and Magnetic Materials, vol. 407, pp. 235-240, 2016.

[31] D. Pal, "Heat and mass transfer in stagnation-point flow towards a stretching surface in the presence of buoyancy force and thermal radiation," Meccanica, vol. 44, no. 2, pp. 145-158, 2009.

[32] M. M. Rashidi, N. Vishnu Ganesh, A. K. Abdul Hakeem, and B. Ganga, "Buoyancy effect on MHD flow of nanofluid over a stretching sheet in the presence of thermal radiation," Journal of Molecular Liquids, vol. 198, pp. 234-238, 2014.

[33] D. Pal and H. Mondal, "Hydromagnetic convective diffusion of species in Darcy-Forchheimer porous medium with nonuniform heat source/sink and variable viscosity," International Communications in Heat and Mass Transfer, vol. 39, no. 7, pp. 913-917, 2012.

[34] Y.-M. Chu, M. I. U. Rehman, M. I. Khan et al., "Transportation of heat and mass transport in hydromagnetic stagnation point flow of Carreau nanomaterial: dual simulations through Runge-Kutta Fehlberg technique," International Communications in Heat and Mass Transfer, vol. 118, Article ID 104858, 2020.

[35] A. V. Kuznetsov and D. A. Nield, "Natural convective boundary-layer flow of a nanofluid past a vertical plate: a revised model," International Journal of Thermal Sciences, vol. 77, pp. 126-129, 2014.

[36] D. A. Nield and A. Bejan, Convection in Porous Media Donald A, Springer, New york, NY, USA, 3rd edition, 2006.

[37] M. Mustafa, J. A. Khan, T. Hayat, and A. Alsaedi, "Buoyancy effects on the MHD nanofluid flow past a vertical surface with chemical reaction and activation energy," International Journal of Heat and Mass Transfer, vol. 108, pp. 1340-1346, 2017.

[38] M. S. Anwar, "Numerical study of transport phenomena in a nanofluid using fractional relaxation times in Buongiorno model," Physica Scripta, vol. 95, no. 3, p. 035211, 2020.

[39] S. Jain and S. Bohra, "Heat and mass transfer over a threedimensional inclined non-linear stretching sheet with convective boundary conditions," Indian Journal of Pure \& Applied Physics, vol. 55, pp. 847-856, 2017. 